\title{
SCLRP - Architecture for Secure Cross-Layer Routing Protocol for Underwater Acoustic Sensor Networks using Fuzzy Logic and Enhanced Algebra Homomorphic Encryption
}

sathiamoorthy jayaraman ( $\nabla$ jsathyam74@gmail.com )

Thiruthangal Nadar College

Usha M

MEASI Institute of Information Technology

Bhagavath Nishanth R

University of Western Sydney: Western Sydney University

Ashween R

Amrita University: Amrita Vishwa Vidyapeetham

\section{Research Article}

Keywords: Quality of service, UASN , Protocol , Fuzzy , Security and encryption, Algebra Homomorphism

Posted Date: April 26th, 2021

DOl: https://doi.org/10.21203/rs.3.rs-227648/v1

License: (c) (1) This work is licensed under a Creative Commons Attribution 4.0 International License.

Read Full License 


\title{
SCLRP - Architecture for Secure Cross-Layer Routing Protocol for Underwater Acoustic Sensor Networks using Fuzzy Logic and Enhanced Algebra Homomorphic Encryption
}

\author{
Dr. J.Sathiamoorthy \\ Associate Professor \\ $T N C$ \\ Chennai, India \\ jsathyam74@gmail.com
}

\author{
R. Bhagavath Nishanth \\ M.S Student \\ West Sydney University \\ Australia \\ nishanth.sachinr@gmail.com
}

\author{
R. Ashween \\ B.Tech Student \\ Amrita Vishwa Vidyapeetham \\ Coimbatore, India \\ ashweenr1998@gmail.com
}

\begin{abstract}
:
The latest research in WSNs (Wireless Sensor Network) has been exploring Underwater Acoustic Sensor Networks (UASNs). As the name suggests, these networks aim at providing effective communication underwater by addressing the challenges prevalent in this network. Underwater communication has found a lot of applications in ocean monitoring and underwater contamination monitoring. The issue of routing underwater is a major concern as it has to address a lot of challenges. Creating an effective routing protocol has been a constant topic of research in UASNs. We have focused on a Secure Cross-Layer Routing Protocol (SCLRP) in UASNs using Fuzzy Logic and Enhanced Algebra Homomorphic Encryption. This protocol aims at providing secure data transmission using optimized nodes that are selected from the neighboring nodes of the source node. The optimal nodes are selected by applying fuzzy rules to the available input. The selected nodes will then forward the packets to the next hop till the packet reaches the destination. The proposed protocol is an Energy Efficient Secure Fuzzy Logic based Cross -Layer design routing protocol. Our proposed protocol ensures safe transmission of data, which is the main objective of a routing protocol used in a wireless network. The routing protocol will ensure better QoS, by using a reliable encryption technique to reinforce secure routing. The protocol incorporates an enhanced Algebra Homomorphic encryption technique to help with managing the security of the transmitted data. QoS is further improved by using a cross-layer design wherein, the proposed protocol will not be expected to adjust as the data travels the different layers of the network so the protocol will be free from the confines of the standards and protocols followed in each of the layer. This flexibility will help in making our protocol perform better than the existing techniques. The SCLRP protocol is analyzed with the performance of NADIR and EEMCCP schemes to analyze its performance.
\end{abstract}

Keywords: Quality of service · UASN · Protocol · Fuzzy · Security and encryption, Algebra Homomorphism,

\section{Introduction}

Wireless Sensor Networks have expanded their technology by exploring communication underwater. Underwater communication is very useful in studying patterns and changes in the ocean's resources. Underwater Communication has found varied applications in developing stable ocean observation systems by deploying the best of what wireless sensor networks have to offer. Applications in underwater communication range from military, oceanography, prediction and warning of underwater calamities Earth is mostly covered in water [1] which has sparked an interest among researchers to explore the various resources that the oceans have to offer. To extend this research, a lot of communication has to be carried out underwater. Underwater Acoustic Sensor Networks (UWASN) have a major role in aiding this observation of the ocean bed for a variety of applications [1][2]. Underwater disturbances and oceanic bed eruptions have compelled scientists to study the principles of underwater communication by deploying sensors wherever necessary. Sensors and Autonomous Underwater Vehicles (AUVs) are the major components that help facilitate underwater communication. However, the aspect of security needs to be strengthened. In fact, the security aspect needs to be addressed without compromising on the quality [3]. Underwater communication is plagued by various challenges like high bit error rates, propagation delay and low channel bandwidth. The mobility of the AUVs poses a greater challenge as they tend to constantly move along with the network. The movement of the sensors and the AUVs are constantly hindered by the strong undercurrents in the ocean. Despite these, communication, and inter-vehicle coordination needs to be maintained at all times for successful transmission throughout the network. The underwater communication network is at maximum risk to malicious external intruders. Efficient and reliable security mechanisms need to be in place to achieve a good coordination among the sensors and their communication points and their counterparts on land [4]. Fig.1(a \& b) shows various underwater communication models. 


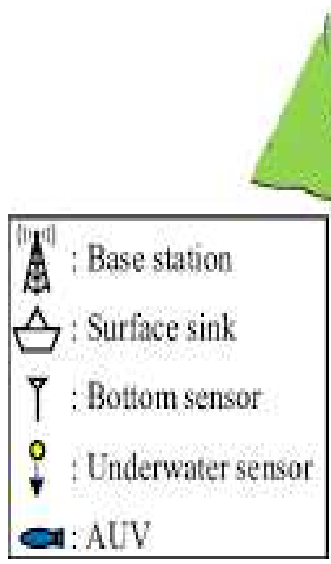

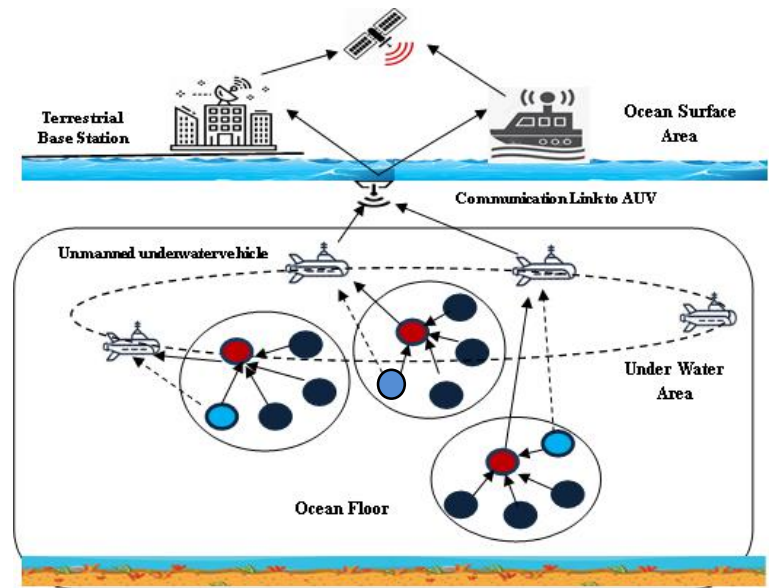

(b)

Fig.1 (a \&b) under water communication models

\section{Related Works}

EEMCCP (Energy Efficient Minimum Cost Cluster Routing Protocol) uses the concept of clusters for routing. The Chaotic Algae Algorithm (CAA) is used to cluster the network. One member node of each cluster is selected to act as the $\mathrm{CH}$ (Cluster Head)[5][6]. The EEMCCP scheme uses only a minimum number of nodes for routing the packets. RSSI (Received Signal Strength Indicator) values are used to select the gateway $\mathrm{CH}$ nodes. These Gateway nodes propagate the processed data to the AUV nodes for further transmission to the surface station. The surface station handles parallel communication with the sink nodes underwater. The base station or the surface station uses the Radio Frequency signals to route the data towards the destination [7].Protocols related to effective routing in Wireless Sensor Ad hoc Networks has always been under research. The routing protocol must incorporate effective and reliable communication as part of its design framework. In fact routing in UASNs is far more challenging than routing for terrestrial regions. The routing protocols for underwater communication face a lot of limitations and restrictions than on land [8]. The movement of the currents underwater poses a challenge to the moving nodes. Also, the propagation of delay underwater is higher than on land. Additionally, UASN based technologies are limited in their operations due to the underwater acoustic waves and channels. Most of the time, underwater acoustic sensor networks are expected to function at short notice. In other words, the communication setup needs to be deployed with no prior arrangement underwater. In case of a broken route, the routing protocol must be able to repair it without undue delay [9]. The routing protocol designed for underwater communication must be self-adaptive and robust at all times. The architectural implementation of UASNs has been under constant research to provide effective solutions for UASNs. The work under research has explored areas where protocols for routing, MAC layer, link layer, communication etc. have been studied thoroughly. The principles and characteristics of different protocols were compared regarding the important aspects such as clustering and transmission [10-12].The protocols were also categorized based on their architecture, data forwarding methods and related operations [12]. This work also reviewed the protocol design of the underwater acoustic network. This work further studied the various research aspects and challenges prevalent in underwater communication (especially, MAC, network and transport layers). The latest research on UASNs has been analyzed by the authors to understand the current challenges and issues in underwater communication[13].Special attention was given to the AMC layer and Network layer as these two need to be well developed to achieve effective communication[14][15]. The linear growth in the number of nodes entering the network may make the network difficult to manage. Clustering is a good solution to manage the network when the network density increases.Clustering also ensures secure and reliable transmission among the trusted nodes in a wireless sensor network. Clustering has proven effective in other ad hoc networks like MANETs, VANETs and FANETs. UWSNs can benefit immensely from implementing a cross-layer design in their protocols. Underwater communication is riddled with a lot of challenges and issues in terms of routing and security. To address these issues, using a cross layer approach would be much more beneficial in terms of not having to worry about standards and protocols exclusive for each layer. This would offer a lot of freedom to the designed protocols in solving the prevalent issues in underwater communication rather than focusing on addressing the protocol standards for each layer. Due to the limited resources, cross-layer optimization will work effectively in combining 
the resources of the layers of the OSI model. The technologies adopted in a cross-layer framework has to focus on the following namely,

- Scheduling: Several node scheduling tasks are taken up in the network. The scheduling technology can be streamlined to accommodate varied networks[16,17]

- Diversified Technology: Diversified technologies are used to adapt to the changing network dynamics and helps improve the reliability of the networks[16][18-20]

- Self -adaptive mechanism: The protocol standards can change dynamically according to the network conditions and topology. The system robustness can be greatly improved when the self -adaptive mechanism coordinates with the scheduling and diversified technologies[21][22]

Encryption has always been combined with the network protocols to ensure secure information [23]. With the help of a secure key, it is possible to transfer data along any type of network. The Ciphertext is very secure and is useless without the correct key. Various encryption techniques have been proposed and implemented by several researchers. These techniques are capable of withstanding any type of unauthorized attacks. Rivest et.al have studied the scope of performing computational operations on encrypted data without decrypting it. This has posed an open problem (known as homomorphic encryption) in the field of cryptography [24]. Fully homomorphic encryption (FHE) allows arbitrary operations on the encrypted data. Researchers have found a way to do multiplication or addition on the encrypted data [25-30]. Recently, schemes have been proposed to conduct both multiplication and addition on encrypted data [31-33]. Gentry et.al have developed an FHE scheme in 2009[34].This scheme is capable of supporting both multiplication and addition of operations without any limit to the number of operations that can be committed. Gentry's FHE is not completely efficient but was able to achieve a moderate amount of success. Various FHE schemes have been proposed to improve on Gentry's proposed scheme [35]. This has helped in improving upon Gentry's initial work [36].

The research work proposed in this paper aims at developing a robust routing protocol that can carry forward the data without consuming a lot of energy. This will help in maximizing the network lifetime to a greater extent. The routing protocol will focus on selecting the next optimal node that will successfully forward the packet to the nexthop node. The next-hop node will be chosen according to the energy levels of the neighboring nodes .The consequent nodes are selected dynamically again based on the energy levels of the surrounding nodes. The proposed work focuses on deploying a cross-layer algorithm. This algorithm utilizes the information gathered from the different layers to assist the protocol responsible for routing to arrive at a decision concerning the next hop. Since the energy of the nodes is not constant, the SCLRP scheme incorporates a fuzzy logic based approach that will help in selecting the next optimal hop.

\section{Motivation}

Previous research on network routing protocols has been following a non- crosslayer design. The network layers, as we know, are categorized into five layers. Each layer has its own set of protocols and standard operations. The interface between the layers is static. Any protocol that is designed works only in the specific layer it is meant for. In fact, these protocols can manage any problems in their layer with the services provided by the lower layer and in turn, they can also provide services to the upper layer. Communication tends to occur only between the adjacent layers. Because these protocols do not support crosslayer optimization, they tend to face issues when they pass through the other layers. Due to their non-compatibility within different layers, they often end up short of their intended objectives. Protocols must be independent of the network layers. For this, the designed protocols need to adapt a crosslayer optimized approach. In fact, cross-layer optimization is simple and easy to implement .robustness and clear and specific logic are the specialties of a protocol that follows cross layer optimization.

The proposed Secure Cross-Layer Routing Protocol (SCLRP) in Underwater Acoustic Sensor Networks using Fuzzy Logic and Enhanced Algebra Homomorphic Encryption aspires to contribute the following

- To transcend the boundaries of the network layers by adapting a cross -Layer optimized approach independent of the standards and protocols specific to each layer.

- To address the prevalent issues common to UASNs

- To develop an effective routing protocol for nodes routing in UASNs

- To secure the data is routed with a strong encryption algorithm

- To enable the selection of an optimized node among the neighboring nodes for efficient routing to the 
next- hop node.

\section{Challenges}

UASNs face a lot of challenging issues .The proposed protocol must be able to address these issues, namely,

- Underwater ocean current which is a hindrance to the mobile nodes

- Disruption in signals due to strong water currents

- Mobility of the nodes interrupted by the underwater ecosystem

- Energy of the node depletes faster

- Security issues both to the node and the data

- Higher propagation delay

- Noise and interference affecting the transmitted signal

- The dynamic topology of the network is constantly affected by the water current

\section{Background of Proposed Routing Protocol Design}

In this paper, we will be focusing on achieving the principles of a Secure Cross-Layer Routing Protocol (SCLRP) design for Underwater Acoustic Sensor Networks. This protocol will incorporate Fuzzy Logic and Enhanced Algebra Homomorphic Encryption into its design. The principles of this protocol will include the following

- Inclusion of the principles of a cross layer optimized approach

- A fuzzy logic based algorithm for the selection of an optimal node (used in routing )

- Secured multipath routing incorporating encryption( for securing the transmitted data)

The Protocol design comprises of two major components, namely,

- A fuzzy controller

- A cross-layer module (to collect parameters from other layers)

These components are implemented in all the nodes to aid the process of routing. Each node in the network is expected to perform two roles, namely,

- Sensing

- Data forwarding

The routing algorithm works with the assumption that every node is surrounded by a group of nodes that are its neighbors. These nodes will help in forwarding the data to the other nodes along the hop route.

\subsection{Principle of Proposed Secure Cross-Layer Design Routing Protocol}

\section{Phase I: Fuzzy Logic Based Routing}

Fig.2 illustrates the architecture of the proposed Secure Cross-Layer Routing Protocol (SCLRP) in UASNs using Fuzzy Logic and Enhanced Algebra Homomorphic Encryption. The cross layer optimization approach adapted in this protocol helps in faster sharing of information. Additionally, the design can be made cost-effective by reducing the overhead usually incurred in communication and signal processing. Using an enhanced Algebra Homomorphic encryption technic, the SCLRP protocol ensures that the transmitted data is safely secured from the perpetrators of the network. To aid the network routing process, SCLP utilizes a Fuzzy approach using a logical controller. This fuzzy approach helps in finding out the best possible neighbor node which will help in forwarding the packets to its next hop node. The main objective of using a cross layer design in SCLRP is to achieve a better network performance. The cross layered approach can achieve the following

- Preserving the layered architecture

- Overcoming the limitations in communication between the different layers

- Enhancing the QoS of the network

A protocol supporting cross layer optimization is capable of adjusting the configuration of parameters specific to each layer. It also increases the amount of information shared in different layers and is also efficient in reducing the cost overheads involved in signal processing and more importantly, communication. In other words, cross 
layer optimization aims at achieving the QoS requirements of WASNs.

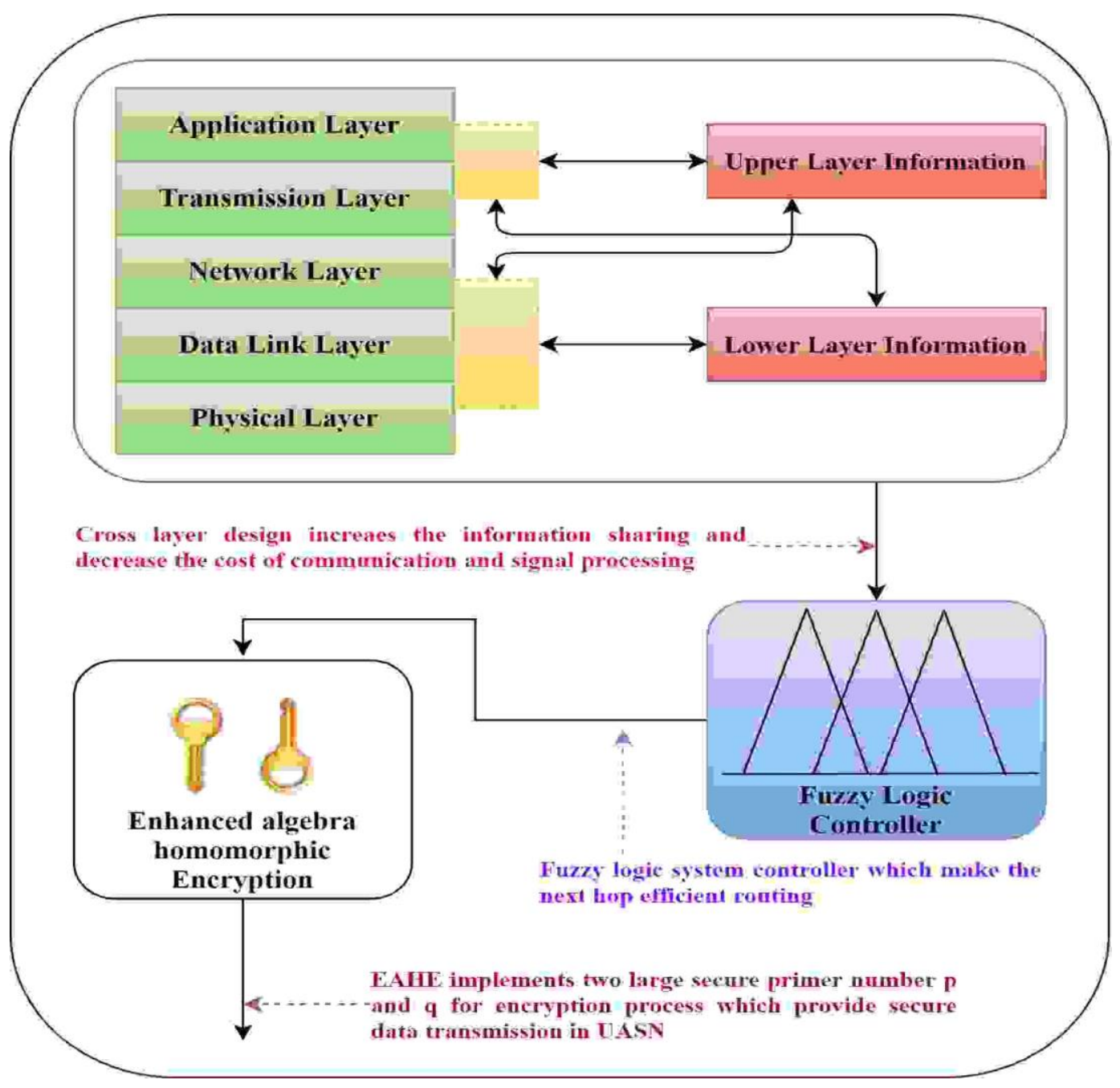

Fig .2 The architecture of proposed secure cross-layer protocol design

Underwater communication is being pursued today because of a variety of reasons. The major reason is to collect data about the changes in the underwater environment. The patterns of the underwater ecosystem are being under study today as they have a major role to play in balancing the life on earth. When a network is established to carry out communication underwater, it starts transmitting messages (either to a communication point underwater or to a terrestrial destination).The nodes involved carry forward the information between the source and the destination. Routing plays a vital role in carrying forward the message packets. When an event occurs underwater, it is recorded and sent to the control center where the acquired data is processed for meaningful information. It is very important to establish an effective and trustworthy route between the source and the destination. Compared to the routing protocols designed for terrestrial networks, the design of the protocols for an underwater environment is much more challenging. UASN requires specific tailor made protocols that encompass the challenges in routing underwater. The practical difficulties of underwater routing need to be addressed while framing a routing protocol for UASNs.

Any protocol incorporating the concept of cross layer optimization needs to maintain the integrity of the functionalities associated with its own layer. The protocol has to ensure the following for the other layers such as

- Coordination

- Interaction

- Joint optimization of protocols 
Adapting a cross-layer approach enhances the compatibility of the architecture to the various layers. This also enables the service to be provide to all the layers at all times. The cross layer approach is still a hot topic of research as scientists are trying to improve the QoS by increasing the efficiency of the approach. The main aim is to improve the optimization of the approach.

The proposed SCLRP routing protocol aims to incorporate the following properties

- The computations involved will not increase in proportion as the constraints increase

- Ability to manipulate the constraints of the cross-layer approach

- Handling the dynamic changes in network conditions and topology(in UASNs)

- Achieving a balance in selecting the next neighboring relay node for routing

The mathematical notations and basic definitions that will be followed in this paper are as follows.

$n$ : Sequence number of the different cross-layer parameters

$i$ : Sequence number of different nodes

$\alpha_{n}$ : Weight of the different cross-layer parameters

$\alpha$ : Weight matrix

$\beta_{n}$ : Cross-layer parameter matrix of parameter $n$

$\beta_{\mathrm{n}}^{\mathrm{i}}$ : Cross-layer parameter $n$ of node $i$

$D_{n}$ : Dispersion of parameter $\beta_{n}$

$D$ : Dispersion matrix

$U^{i}:$ Utility of node $i$

' $\alpha$ ' is a weight matrix. It represents the various cross-layer parameter weights . $\alpha$ is represented in equation(1)

$$
\propto=\left[\propto_{1}, \propto_{2}, \propto_{3}, \ldots, \propto_{n}\right]
$$

The cross layer parameter matrix consists a set of cross-layer parameters represented by $\beta_{\mathrm{n}}$ (obtained from node i). $\beta_{\mathrm{n}}$ is expressed in equation(2)

$$
\beta_{n}=\left[\beta_{n}^{1}, \beta_{n}^{2}, \beta_{n,}^{3}, \ldots \ldots, \beta_{n}^{i}\right]^{T}
$$

The various cross layer parameters that can be considered are

- The number of hops and the time taken to reach the base station

- Encountered link breaks along the route

- Interface queue length

- Residual battery power

- Number of hops towards the destination node

The scatter of the different parameters of the cross layer is decided by using the concept of dispersion (denoted by the dispersion matrix D). The mean of the cross -layer parameters of the various nodes is given by $\overline{\beta \mathrm{n}} . D_{n}$ and $D$ is expressed in equations (3) and (4).

$$
D_{n}=\frac{\left.\left|\beta_{n}^{1}-\overline{\beta_{n}}\right|+\left|\beta_{n}^{2}-\overline{\beta_{n}}\right|+\left|\beta_{n}^{3}-\overline{\beta_{n}}\right|+\cdots+\left|\beta_{n}^{i}-\overline{\beta_{n}}\right|\right) / i}{\overline{\beta_{n}}}
$$




$$
D=\left[D_{1}, D_{2}, D_{3} \ldots, D_{n}\right]^{T}
$$

The order of the next hop relay node from a set of neighboring nodes is decided by the utility of node i. This is expressed in equation (5)

$$
U^{i}=\propto * \beta_{n}
$$

The main objective of the SCLRP scheme is to calculate the value of $U^{i}$. The value of $U^{i}$ is then used to select the best optimal next hop relay node from the neighbor nodes.

\subsubsection{Calculation of the Utility of Each Node}

As stated above the utility value decides the order of the next hop relay nodes. This ensures that the packet is forwarded by the optimal nodes to achieve the objective of routing. Equation (5) can be applied to calculate the utility value after obtaining the weights for the nodes (after processing).

The utility value of node $i$ is expressed in equation (6):

$$
U^{i}=\left[\propto_{1} \beta_{1}^{i}+\alpha_{2} \beta_{2}^{i}+\cdots+\alpha_{n} \beta_{n}^{i}\right]
$$

Where

$\beta_{\mathrm{n}}^{\mathrm{i}}-$ denotes cross-layer parameters of node $i$

$\alpha_{n}$ - various parameter weights

The major work of the source node is to gather the parameters of the cross-layer (denoted by $\beta \mathrm{n}$ ) from the set of neighboring nodes. The source node duly determines the value of dispersion of each parameter (denoted by $D_{n}$ ). The fuzzy logic inference system is used by the source node to input the values of Dn to the inference engine. This value will be instrumental in determining the weights $\alpha_{\mathrm{n}}$. The values of $\alpha_{\mathrm{n}}$ and $\beta_{\mathrm{n}}$ are used to determine the utility value of each node. This utility value will help in selecting the optimal neighbor node, which will act as the next hop relay node in forwarding the data packet. Fig. 3 represents the process involved in determining the utility value of a node from a set of neighboring nodes based on the values provided to the fuzzy inference system.

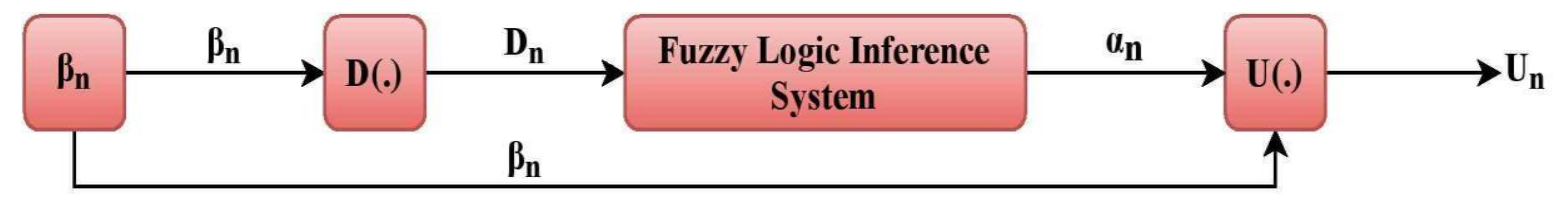

Fig. 3 The principle of the SCFL

\subsubsection{Fuzzy Controller Design}

The proposed SCLRP scheme uses three main parameters namely,

- The neighboring nodes' battery power

- Signal strength reception by the node

- Transmission status of the node

These parameters help the routing algorithm in deciding on a decision about the selection of the next hop relay node for forwarding. The nodes within a network exchange energy information among themselves. This information is made available to the other nodes by using the technique of piggybacking. Every node maintains a linked list containing the residual energy levels of its neighboring nodes. The parameters thus collected by every node (in a localized manner) serves as the input to the fuzzy logic controller. The output from the fuzzy logic controller comprises of the probability of selecting a neighbor node as the next routing node. The fuzzy logic controller fast forwards the decision making process by conceding between significance and precision.

\subsubsection{The Primary Elements of a fuzzy logic controller are}


- The Fuzzifier

- Inference engine

- The fuzzy rule base

- The Defuzzifier

The functions of these elements in the proposed algorithm are detailed in the following sections. (Fig. 3) shows the basic set of a fuzzy inference engine.

\subsubsection{Fuzzification of the input variables}

The proposed algorithm uses the following parameters as inputs

- Remaining battery level

- Reception of signal strength

- Level of energy for transmission

The inputs supplied to the fuzzy interference engine are mapped on to the suitable fuzzy sets. The altered input variables thus obtained is the Relative Energy Level (REL) of a node. REL is the factor that defines the residual energy level of a node with respect to their neighborhood location. REL is calculated using

$$
R E L=\frac{E_{\text {node }}-E_{\text {min }}}{E_{\text {max }}-E_{\text {min }}}
$$

where,

Emax, Emin - high and low energy levels of the nodes in the vicinity.

$\mathrm{E}_{\text {node }}$ - residual node energy

The value of REL defines the criticality of a node. The higher the value of REL, the lesser the criticality of a node we use Frii's transmission equation to compute REL This equation uses a power relation and is given as

$$
P_{R x}=P_{T x} G_{t} G_{r}\left(\frac{\lambda}{4 \pi d}\right)^{2}
$$

where,

$\mathrm{P}_{\mathrm{Rx}}$ - received power

$\mathrm{P}_{\mathrm{Tx}}-$ communication strength

$\Lambda$ - Signal wavelength

$\mathrm{d}$ - Distance between the sender and the receiver

$\mathrm{G}_{\mathrm{t}}$ and $\mathrm{G}_{\mathrm{r}}$ - the antenna gain of the transmitter and the receiver.

\subsubsection{Membership functions}

The fuzzy inference controller uses triangular membership functions for each of the input and output. This simplifies the whole process and makes it easier to implement. Choosing a suitable member function aids in achieving the desired results. Rule evaluation occurs when Fuzzifier inputs are supplied to the system, which are later applied to antecedents of the fuzzy rules. This is then applied to the resultant membership functions. The rule based structure of the fuzzy logic is used to formulate a series of " IF X AND Y THEN Z " rules. These rules are defined to structure the response of the system to the input conditions supplied to them. Table 1 illustrates the proposed fuzzy rules. Figures represent the membership functions defined for the input and the output.

\begin{tabular}{|c|c|c|c|c|c|c|c|}
\hline $\begin{array}{c}\text { Input } \\
\left(\mathrm{D}_{n}\right)\end{array}$ & $\begin{array}{c}\text { Very } \\
\text { small }\end{array}$ & $\begin{array}{c}\text { Medium } \\
\text { small }\end{array}$ & Small & Medium & Large & $\begin{array}{c}\text { Medium } \\
\text { L arge }\end{array}$ & $\begin{array}{c}\text { Very } \\
\text { Large }\end{array}$ \\
\hline $\begin{array}{c}\text { Output } \\
\left(\alpha_{s}\right)\end{array}$ & $\begin{array}{c}\text { Very } \\
\text { Large }\end{array}$ & $\begin{array}{c}\text { Medium } \\
\text { Large }\end{array}$ & Large & Medium & Small & $\begin{array}{c}\text { Medium } \\
\text { small }\end{array}$ & $\begin{array}{c}\text { Very } \\
\text { small }\end{array}$ \\
\hline
\end{tabular}


Table 1. The fuzzy if-then rules of BCFL

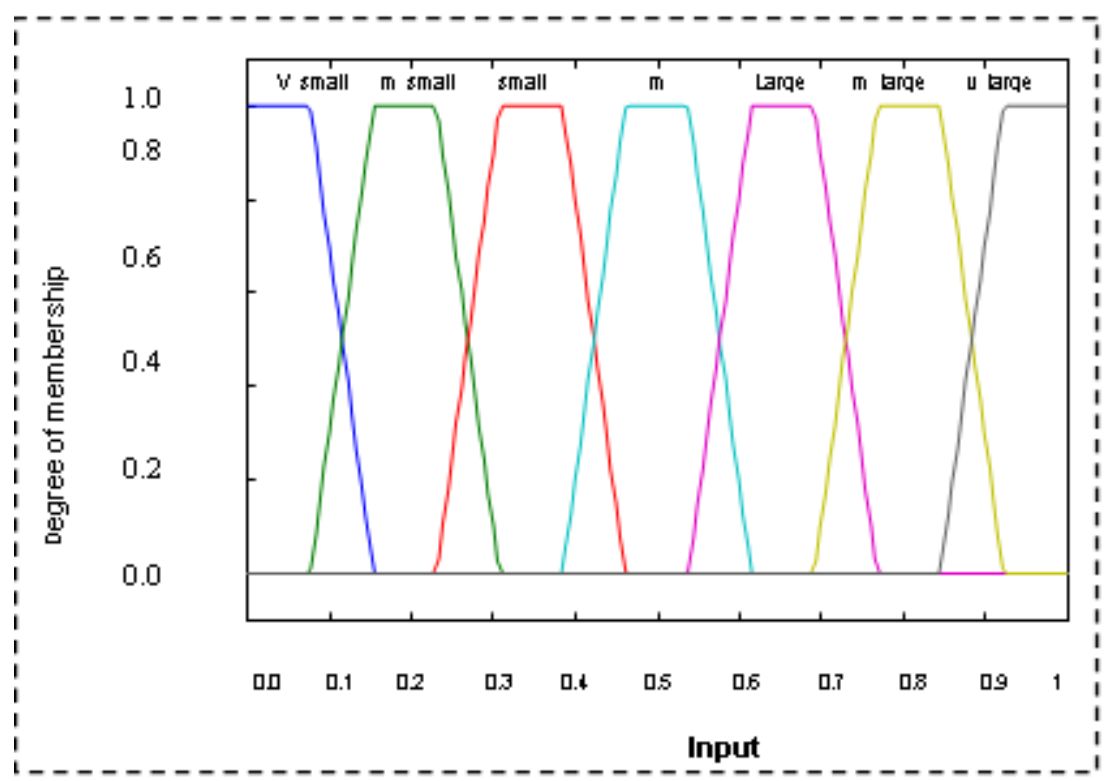

Fig.4 The membership function of input

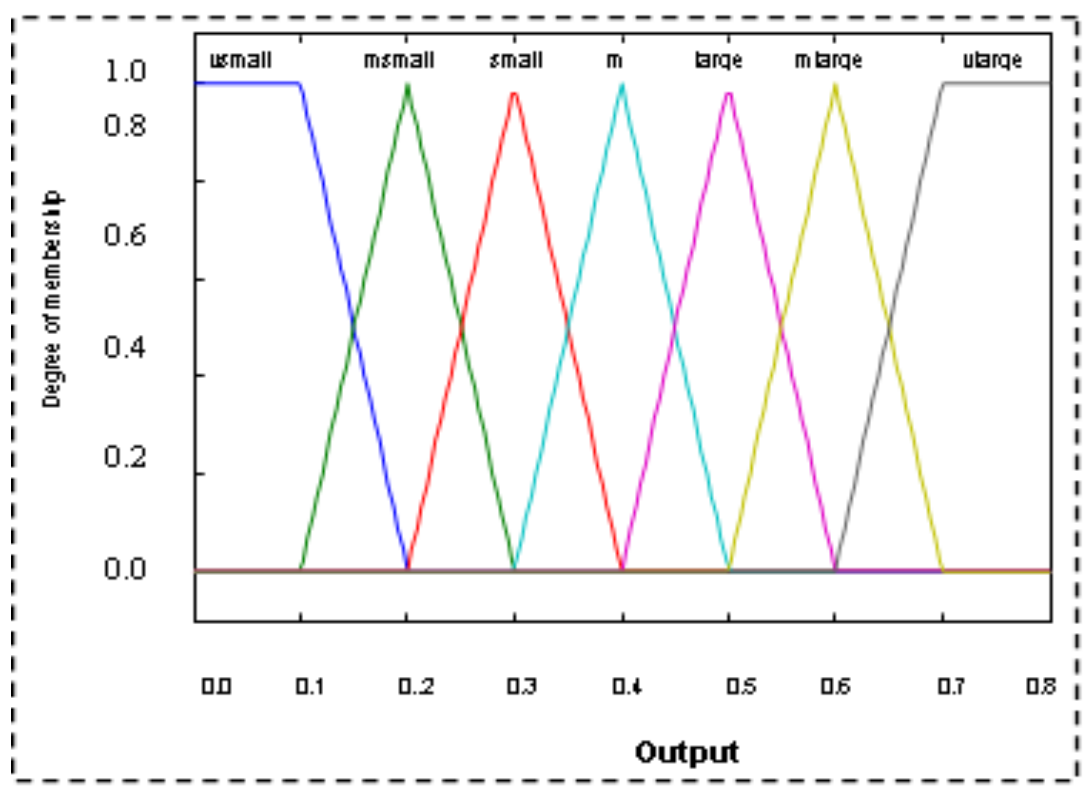

Fig.5 The membership function of output

\subsubsection{Fuzzy Inference Engine}

The role of the fuzzy inference engine is to aggregate the rule outputs. The process of unification is achieved with the help of the weights of the calculated rules and the Max -Min interference methods.

\section{Defuzzification and Fuzzy Control}


The defuzzification process takes the aggregated output fuzzy set as its input. The output thus obtained is a single crisp number. The fuzzy response $P_{f}$ is computed using the centroid method. Fig.4 and Fig.5 represent the membership functions for input and output, while Fig.6 illustrates the fuzzy interference engine.

$$
P_{f}=\frac{\sum_{i=1}^{n} \mu\left(k_{i}\right) * k_{i}}{\sum_{i=1}^{n} \mu\left(k_{i}\right)}
$$

Where,

$\mathrm{n}$ - number of rules activated

$\mathrm{k}_{\mathrm{i}}-$ activated output consequent

$\sum_{i=1}^{n} \mu\left(k_{i}\right) \quad$-The corresponding Rule Degree of the Membership Value.

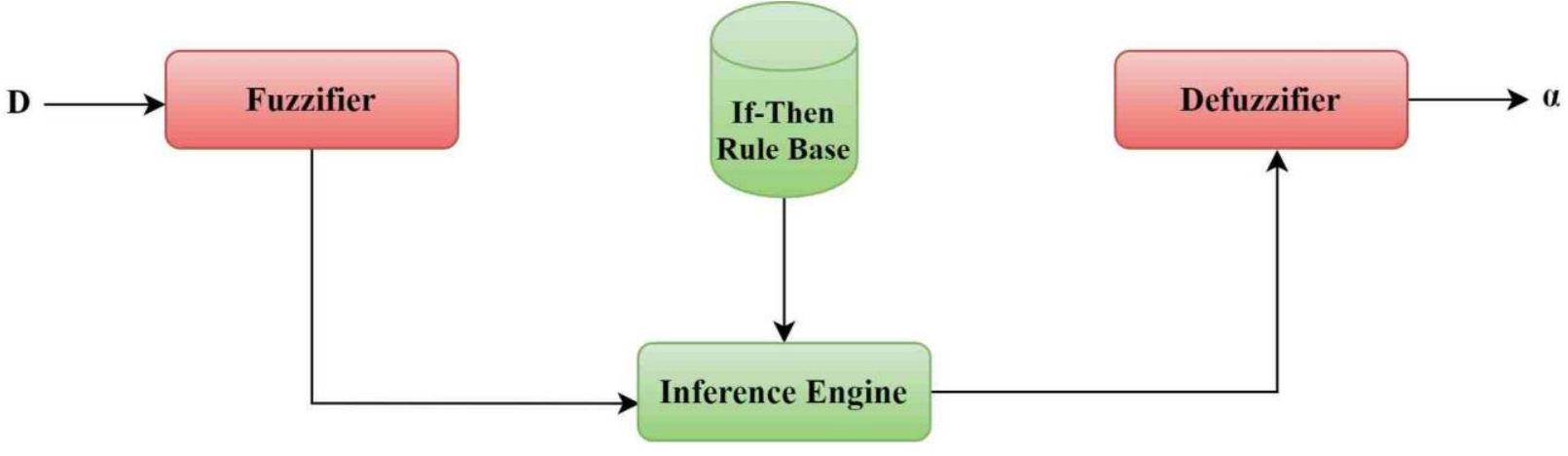

Fig.6 The Principle of Fuzzy Inference System

Algorithm 1 explains the secure cross layer routing protocol the notations used in the algorithm are detailed below. The energy levels of the nodes are broadcast before starting the actual process of routing. This broadcast allows the nodes to store the energy values and the cross layer parameters. If the cross layer parameter of a node meets the necessary threshold criteria, that node is chosen as one of the optimal node .The dispersion values of the nodes is also calculated. The values are fed into a fuzzy inference engine as input to obtain the utility value of each node selected as the optimal nodes. The utility values are ranked and the one that tops the list is identified as the next hop node. This is repeated for all the nodes along the route. In case the selected node is broken or moves away, the next node which has the second highest utility value is automatically chosen without delay. Finding the best optimal node for forwarding the packets helps the SCLRP protocol to find the best possible short route towards the destination. The utility value is calculated when the packet reaches the next node in the subsequent hops. The SCLRP technique has been designed dynamically since there is a possibility of the node moving away before the packet could reach it. Finding a route beforehand is not feasible as the network is formed ad hoc and the nodes tend to move away abruptly. The SCLRP scheme has the ability to find the shortest cost effective route towards the destination node with the assistance of the optimal nodes.

Algorithm 1: Secure Cross-Layer Routing Algorithm (SCLR)

$$
\begin{aligned}
& \beta_{n}^{i}-\text { Cross layer parameters from Node } \\
& \beta_{\text {threshold }}-\text { Cross layer parameter Threshold value }
\end{aligned}
$$$$
N_{\text {Rank }}-\text { Rank of the neighbour node to be the next hop relay node }
$$$$
\text { fun.Dis }\left(\beta_{n}^{i}\right) \text {-Function to compute dispersion of the cross layer parameters }
$$ 


\section{fun. fuzzy $\left(D_{n}\right)$ - Function fuzzy to compute weight of each cross layer parameters}

fun. Utility $\left(\beta_{n}^{i}, \alpha_{n}\right)$ - Function to compute utility of each and every node

fun. Probab $\left(U_{n}\right)$-Identify the rank of the neighbour node to be the next hop relay node

fun. Broadcast(hello Msg) - Broadcast hello message to neighbour nodes

route_dis - Route discovery value

Node_Status - Status of each node in the network

Step 1: Start

Step 2: $\quad$ Set route_dis $=0$

Set Node_Status $=$ false

Step 3: While (route_dis $=1$ ) Do

Step 4: Call fun. Broadcast ();

Step 5: $\quad$ Store the cross layer parameter which is received from other nodes

Step 6: If $\quad\left(\beta_{n}^{i} \geq \beta_{\text {threshold }}\right)$

Step 7: $\quad$ Node_Status $=$ true

Step 8: Else

Step 9: $\quad$ Node_Status $=$ false

Step 10: End If

Step 11: When (Node_Status $==$ true ) then Do

Step 12: $\quad$ Call the below functions order wise for route the data

$$
\begin{aligned}
& D_{n} \leftarrow \text { fun.Dis }\left(\beta_{n}^{i}\right) \\
& \propto_{n} \leftarrow \text { fun.fuzzy }\left(D_{n}\right) \\
& U_{n} \leftarrow \text { fun.Utility }\left(\beta_{n}^{i} \alpha_{n}\right) \\
& N_{\text {Rank }} \leftarrow \text { fun.Probab }\left(U_{n}\right)
\end{aligned}
$$

Step 13: End Do

Step 14: End While

Step 15: Stop 


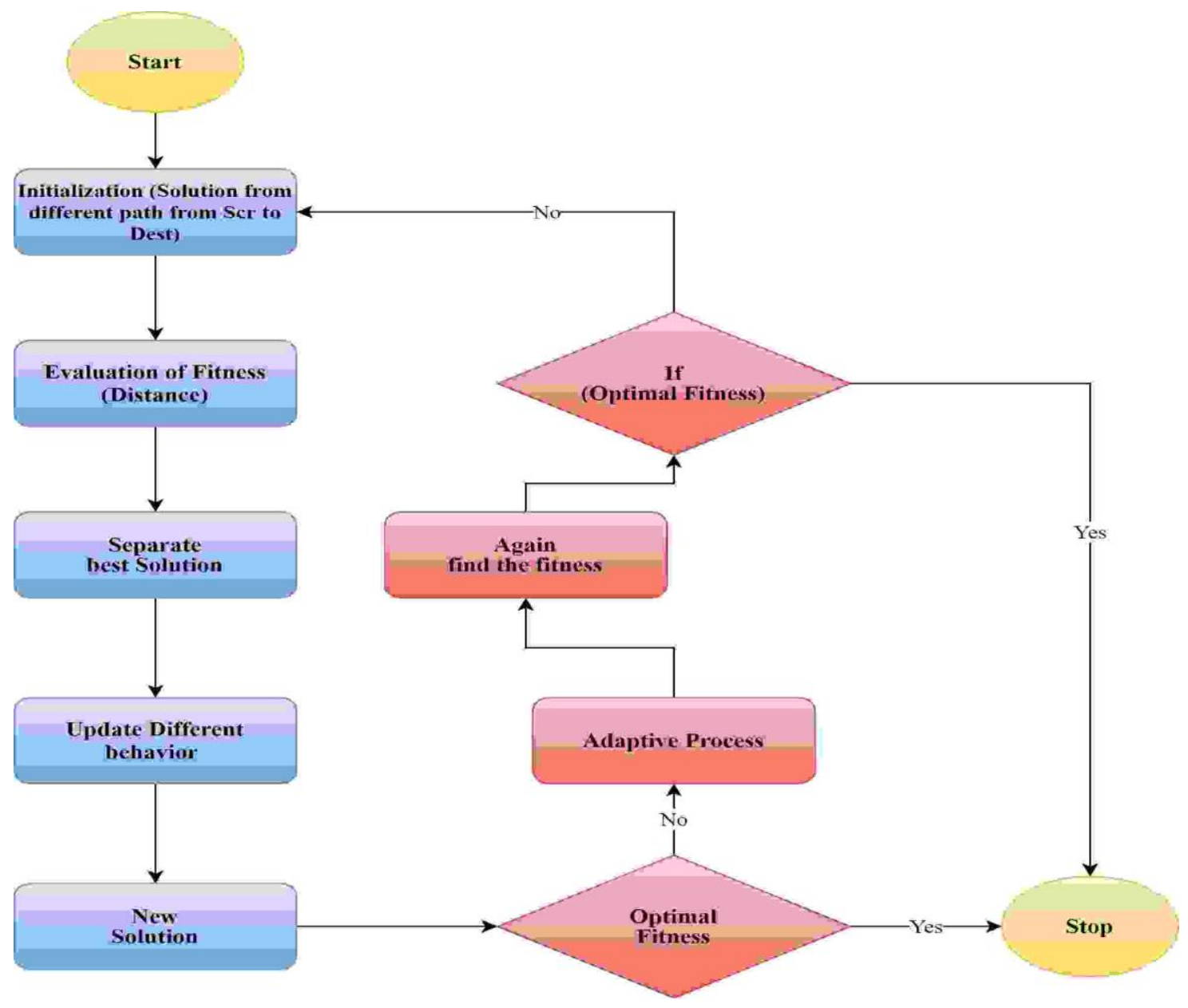

Fig.7 Data Flow Diagram for Efficient Route

Fig.7 depicts the data flow diagram for efficient routing. As explained above, the SCLRP scheme finds the node with the best optimal fitness to route the packet. As this process is carried out, constant updation is carried out for the energy levels and dispersion values of all nodes. These values are used in the utility value calculation.

\subsection{Phase II: Enriched Encryption Process Based on Secure Data Transmission}

The second phase of the SCLRP protocol aims to secure the data that is being transmitted. Encryption is the best way to protect the transmitted data especially when the user wants to prevent unauthorized access to the data. The strength of an encryption scheme relies on the selection of a suitable key to both encrypt and decrypt data. In our proposed scheme we have utilized an Enhanced Algebra Homomorphic Encryption (EAHE) scheme to safeguard the data .This EAHE technique will be very useful as it will provide multi-level security in underwater acoustic networks. The data will be subjected to a strong cryptographic hash function and a suitable encryption algorithm will be used for data encryption with a secure and strong key. This key will be alternatively used in the decryption process to decode the data.

\subsubsection{Enhanced Algebra Homomorphic Encryption (EAHE)}

The main aim of using the Enhanced Algebra Homomorphic Encryption (EAHE) is to allow any user to use the encrypted data to perform important computations and operations without access to the encryption key. This scheme has been applied to several applications especially to improve cloud computing security. If a user wishes to store encrypted sensitive information in a cloud setup but not sure of the cloud service provider, the user can still access and manipulate his data. This scheme also prevents any hacker or external applications from gaining access to the data while the user works on it. The structures of the EAHE are applied extensively to decide on the key to be used 
for the encryption process. The user can ensure perfect safety for his data with a secure key. The private and public key mechanisms are exploited fully to carry out the encryption. The EAHE encryption process consists of the following steps.

The mathematical notations used in the Enhanced Algebra Homomorphism Encryption Scheme (EAHE) are as follows:

Step 1: consider two large secure prime numbers say, $\mathrm{p}$ and $\mathrm{q}$ and $\mathrm{N}=\mathrm{p} * \mathrm{q}$, where, $\mathrm{p}$ and $\mathrm{q}$ are confidential while $\mathrm{N}$ is made public

Step 2: Select a random value $\mathrm{x}$ and a root $\mathrm{g}$ of GF (p). Both $\mathrm{g}$ and $\mathrm{x}$ must be smaller than $\mathrm{p}$. We use the following calculation

$$
Y=g^{x} \bmod p
$$

Step 3: A random positive integer number $r$ is selected. The plaintext $M$ goes through a homomorphic encryption.

$$
E_{t}(M)=(M+r * p) \bmod N
$$

Step 4: An encryption process is applied after selecting a random integer $\mathrm{k}$, given by

$$
E_{g}(M)=(a, b)=\left(g^{k} \bmod p, y^{k} E_{I}(M) \bmod p\right)
$$

Step 5: The decryption process $\mathrm{D}_{\mathrm{g}}()$ is given by

$$
M=b \times\left(a^{x}\right)^{-1}(\bmod p)
$$

Multiplicative homomorphism: When there are plaintext messages $M_{1}$ and $M_{2}, E_{g}\left(M_{1}\right) \cdot E_{g}\left(M_{2}\right)=\left(a_{1} a_{2}, b_{1} b_{2}\right)$ defined, EAHE exhibits multiplicative homomorphism namely,

$$
E_{g}\left(M_{1} M_{2}\right)=E_{g}\left(M_{1}\right) \cdot E_{g}\left(M_{2}\right) \text { or } M_{1} M_{2}=D_{g}\left(E_{g}\left(M_{1}\right) \cdot E_{g}\left(M_{2}\right)\right)
$$

Additive homomorphism: When there are plaintext messages $\mathrm{M}_{1}$ and $\mathrm{M}_{2}, \mathrm{E}_{\mathrm{g}}\left(\mathrm{M}_{1}\right) \mathrm{XOR} \mathrm{E}_{\mathrm{g}}\left(\mathrm{M}_{2}\right)=\left(\mathrm{a}, \mathrm{b}_{1}+\mathrm{b}_{2}\right)$ defined, EAHE is set to exhibit additive homomorphism, namely,

$$
E_{g}\left(M_{1}+M_{2}\right)=E_{g}\left(M_{1}\right) \text { XOR } E_{g}\left(M_{2}\right) \text { or } M_{1}+M_{2}=D_{g}\left(E_{g}\left(M_{1}\right) \text { XOR } E_{g}\left(M_{2}\right)\right)
$$

EAHE exhibits several useful properties because of the selection of the two random numbers. The numbers $\mathrm{k}$ and $\mathrm{r}$ are used in the encryption process. The best part of EAHE is that the results are different for two encryptions of the same plain text. In other words, $\mathrm{E} 1(\mathrm{x}) \neq \mathrm{E} 2(\mathrm{x})$, Also, the decryption is $\mathrm{D}(\mathrm{E} 1(\mathrm{x}))=\mathrm{D}(\mathrm{E} 2(\mathrm{x}))$. This property prevents malicious users from finding out the original data by using statistical measures. In fact, additive homomorphism property of the EAHE technique uses the same k to encrypt but, the introduction of the random number $\mathrm{k}$ in EI () helps the EAHE algorithm resist plaintext attacks. EAHE has been used in critical security based applications involving secure multi-party communication. It has also found varied uses in ad hoc networks like MANETs and underwater communications. The EAHE technique is combined with the SCLRP protocol to provide enhanced security to the transmitted data against malicious users of the networks. Using its unique properties EAHE is used intelligently by the SCLRP scheme to strengthen the security aspect of the data to be forwarded towards the destination. Algorithm 2 explains the process of the EAHE scheme. The notations used int eh algorithm are detailed for the understanding of the algorithm.

Algorithm 2: Enhanced Algebra Homomorphism Encryption Scheme (EAHE)

$\mathrm{p}$ and $\mathrm{q}$ - confidential prime numbers 


$$
\begin{aligned}
& x-\text { random number } \\
& r \text { and } k \text { - integer numbers } \\
& E_{g}() \text { - encryption function } \\
& D_{g}()-\text { Decryption function }
\end{aligned}
$$

Step 1: Start

Step 2: select two data $\mathrm{p} \& \mathrm{q}$ for finding ' $\mathrm{N}$ '

Step 3: If $((\mathrm{p}>0) \& \&(\mathrm{q}>0))$ then

Step 4: Compute $\mathrm{N}=\mathrm{p}^{*} \mathrm{q} / / \mathrm{C}^{\mathrm{N}} \mathrm{N}$ - public

Step 5: $\quad$ If $((\mathrm{g}<\mathrm{p}) \&(\mathrm{x}<\mathrm{p}))$

Step 6: $\quad$ select value ' $x$ ' and a root ' $g$ ' of GF (p)

Step 7: $\quad$ Compute $Y=g^{x} \bmod p / /$ use this ' $y$ ' for encryption process

Step 8: $\quad$ Two steps are required for performing encryption process

Step 9: $\quad E_{t}(M)=(M+r * p) \bmod N / /$ homomorphic encryption

Step 10: $\quad E_{g}(M)=(a, b)=\left(g^{k} \bmod p, y^{k} E_{I}(M) \bmod p\right)$

Step 11: $\quad$ Performing Decryption process $\mathrm{D}_{\mathrm{g}}()$

Step 12: $\quad M=b X\left(a^{x}\right)^{-1}(\bmod p)$

Step 13: $\quad$ Else

Step 14: $\quad$ Goto Step 1

Step 15: $\quad$ End If

Step 16: Else

Step 17: $\quad$ Goto Step 1

Step 18: End If

Step 19: Stop

As stated in the mathematical discussion, the private and the public keys are selected and applied to the plain text to arrive at the encrypted text. The encrypted text can then be used in any computation without the need to encrypt $i$. The decryption is carried out by using the keys known only to the sender and receiver. The EAHE scheme protects the data and at the same time allows the authorized user to work with the original data without the need to decrypt it.

\section{Results and Discussion}

Table 2 Simulation parameters

\begin{tabular}{ll}
\hline Parameters & Values \\
\hline Number of nodes & $25,50,75,100,125$ \\
Packet size & 70 bytes \\
Underwater Area & $1500 \mathrm{~m} \mathrm{X} \mathrm{2000m}$ \\
Transmission Range & $120 \mathrm{~m}$
\end{tabular}


Distance b/w Sensor Nodes

Initial Energy

Data packet Generation by Node

Idle Power

Number of Sink

Simulation time (Sec)
$200 \mathrm{~m}$

70 joule

Every $25 \mathrm{sec}$

0.008

More than 1

300

Table 2 represents the simulation parameters used for the analysis of the proposed technique.The proposed SCLRP

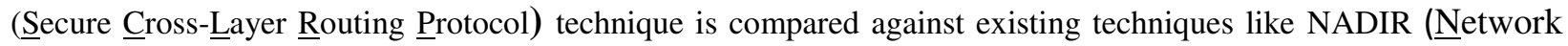

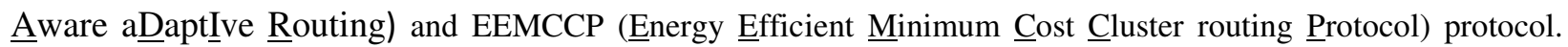
NADIR and EEMCCP are selected because they have both been designed for underwater communication networks. This comparison helps us to study the performance of our proposed protocol over the existing protocols. The comparison is carried out with the help of the Aqua Sim for NS2 version 2.30 simulator. The comparison is based on major network metrics like

- Energy consumption

- Throughput

- $\quad$ End - to -End Delay

- $\quad$ Packet Delivery Ratio (PDR)

The protocols in the graphs have been color coded for better understanding.

SCLRP

- Focuses on energy conservation

- $\quad$ Routing is done by calculating the utility value of all the neighboring nodes using fuzzy rules

- The forwarding relay node is selected using a utility value

- The data is secured using an EAHE algorithm

NADIR

- Focuses on energy conservation

- Works in two phases

- $\quad$ Phase I - sends control messages

- $\quad$ Phase II - routing of messages takes place

-control messages exchanged again indicating the estimated number of hops and link quality

-Residual energy of the nodes is also relayed

-Information sued to select the next relay node

- Encryption techniques are not used

\section{EEMCCP}

- Focuses on energy conservation

- A two phase protocol 
- Phase I - Organizes network into clusters and selects a cluster head

- $\quad-$ Selects a backup $\mathrm{CH}($ Cluster Head)

- Phase II - selection of a gateway nodes to transfer the processed information to the moving nodes

- Encryption techniques are not used

The values supplied for each parameter has been modified from low to high values to study the protocols better.

\subsection{Energy}

Energy consumption is a major issue since the nodes constantly move while transmitting messages underwater. The protocols designed for underwater communication need to be designed in such a way that the energy is conserved at every stage till the data is completely transmitted towards the destination. The protocols' energy consumption levels are studied and we find that the proposed SCLRP scheme exhibits lesser energy consumption than the other two techniques. EEMCCP shows lesser energy conservation than the other two protocols (Fig.8). EEMCCP scheme spends considerable time in clustering the network and then selecting a cluster head. Also, a backup cluster head is selected. Additionally, a gateway cluster head is selected to transfer information to the mobile nodes .All of these tasks drain the nodes of their energy and pose the risk of losing their battery power. All of this plays a major role in minimizing the energy conservation levels of the nodes in the entire network. NADIR has a better energy conservation level than EEMCCP but loses out to the proposed SCLRP scheme. NADIR focuses on routing and spends most of its time sending out control messages informing all the other nodes about several aspects in the network. These control messages are mostly sent via the piggybacking technique. Also, these control messages are sent in both phases and the operations of the protocol rely heavily on the information carried by these control messages. This too drains the energy level of the nodes as they focus heavily on sending out the control messages in selecting the next relay node. The proposed SCLRP scheme uses fuzzy rules to decide the next hop relay node and quickly determines the optimal relay to a node to carry forward the data. This helps is finding the shortest effective route towards the destination and also helps in conserving energy. The SCLRP scheme thus conserves lesser energy in carrying out the operations when compared to NADIR and EEMCCP.

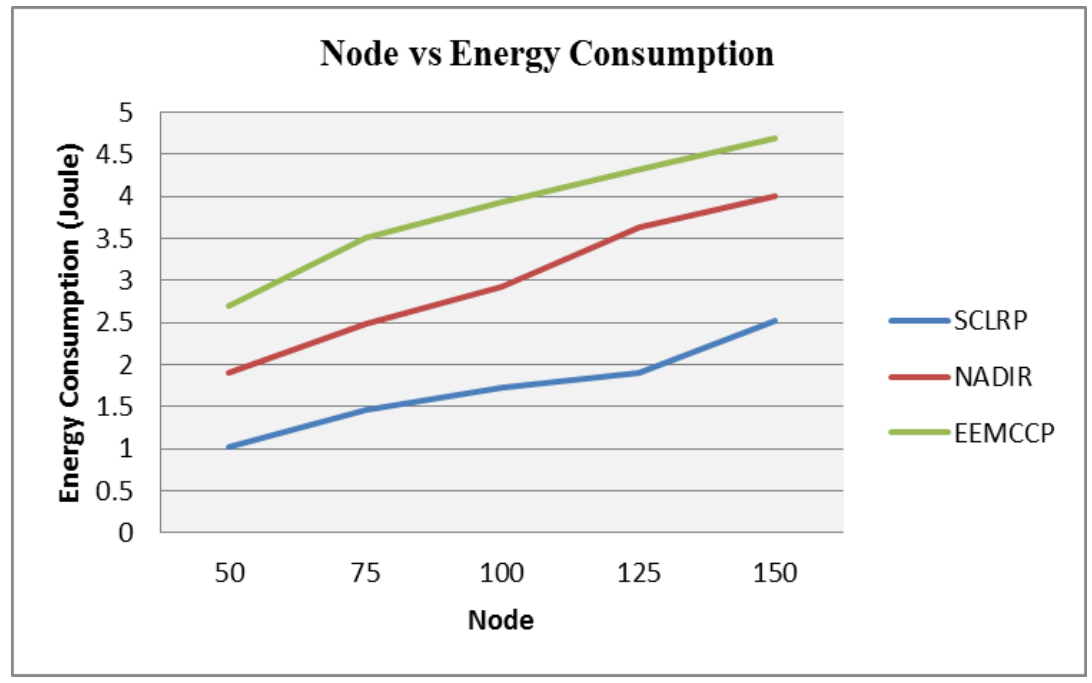

Fig.8 Energy Consumption

\subsection{Delay}

Fig.9 depicts the delay of SCLRP which is lesser than NADIR and EEMCCP schemes. From (Fig.9) we can see that EEMCCP has a greater delay than the other two schemes .The EEMCCP scheme spends its time in clustering and identifying efficient $\mathrm{CHs}$. When a transmission starts and a cluster head moves away from the cluster, it becomes mandatory to select another cluster head. Though a backup cluster head is available, time is lost in appointing the new cluster head and to resume transmission. Again, EEMCCP does not have any encryption schemes to secure the transmitted data. When the network is under attack, the data transmitted may be lost or hacked into. This may result in retransmissions and rearranging the routes, again resulting in losing time as well as bandwidth. This delay plays 
an adverse role in affecting the network throughput. NADIR selects the next relay node for routing the data but it does not use any fuzzy rules like SCLRP. Again, NADIR does not deploy any encryption schemes as part of its operational scheme. Any attack on the data leaves it unguarded and leads to unnecessary delay. This also results in stagnation of the transmitted data leading to a series of related delays. The overall delay is automatically enhanced. The SCLRP protocol applies fuzzy rules to choose the optimal next hop relay node for effective routing. Even if the nodes move away, the node with the second highest utility value is automatically selected as the forwarding node. It also uses an EAHE scheme to safeguard the data against hackers or external attacks. These factors help the SCLRP technique to reduce the delay considerably than the two schemes.

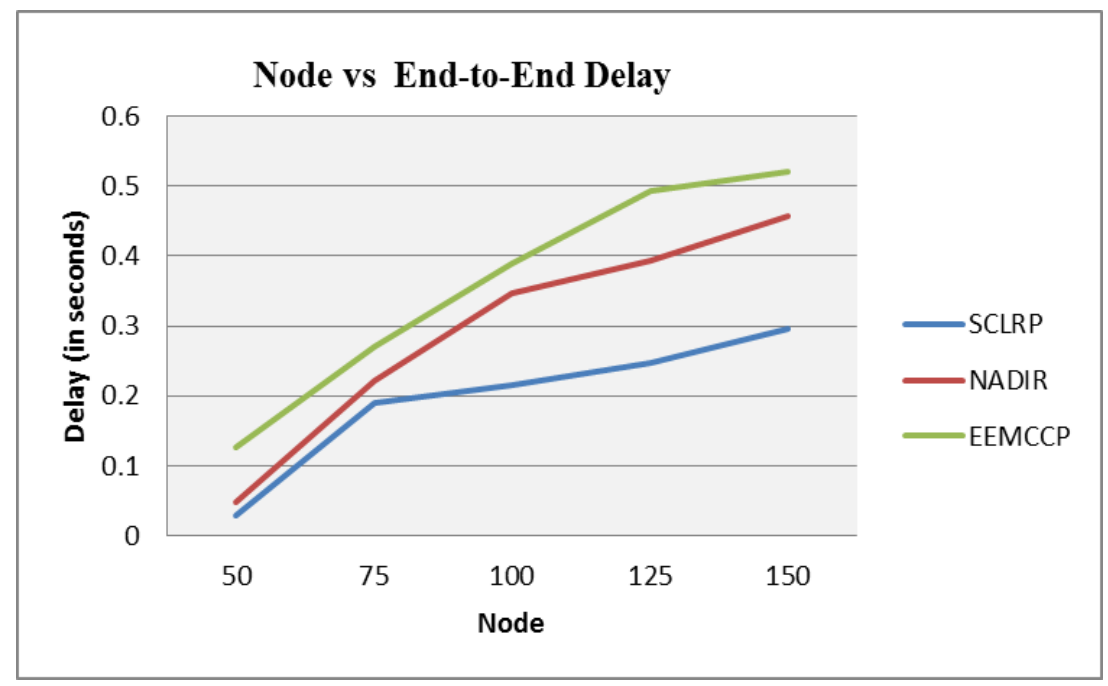

Fig.9 End to End Delay

\subsection{Packet Delivery Ratio}

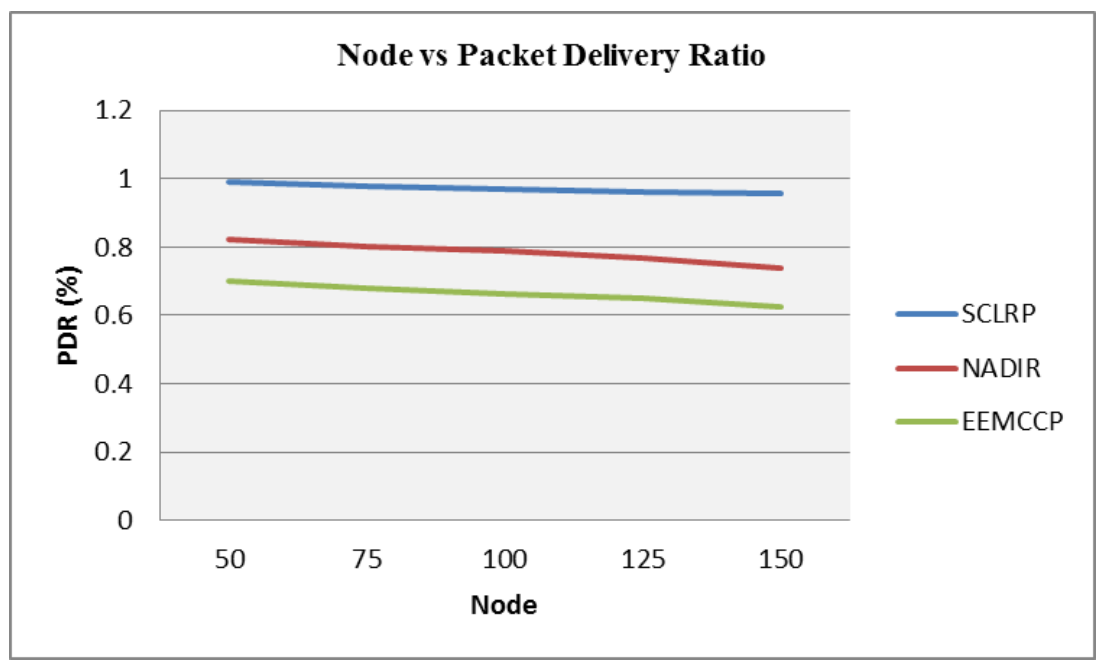

Fig.10 Packet Delivery Ratio

Fig.10 depicts the PDR of a network is determined by both internal and external factors that act upon it. The SCLRP scheme has an effective PDR when compared to NADIR and EEMCCP schemes. The EEMCCP scheme as already stated does not have a strong encryption scheme to secure the transmitted data. Any attack on the data will lead to data loss causing drops or discards of the data packets. This will definitely reflect poorly on the PDR of the EEMCCP technique. NADIR needs time to collect information from the control packets in both of its phases. The time spent on updating the node status can be effectively spent in transmitting the data. Additionally, NADIR does 
not use any secure schemes to protect the transmitted data. Here again, the packets may get lost or discarded due to external attacks. This will affect the overall PDR of NADIR. The proposed SCLRP scheme utilizes fuzzy rules to its advantage by selecting optimal nodes to forward the data. While the data is being transmitted, it is protected from unauthorized access by using a strong encryption scheme like EAHE. The packets transmitted may get lost only due to other network factors but, there is a high chance that the packets will be secure and will reach the destination. This can be attributed to the fact that the nodes along the route are selected based on their utility value. As a result, the PDR of SCLRP is maximized than the other two schemes.

\subsection{Throughput}

The overall performance of any network is determined by the various factors that govern the protocols that work on it. The proposed SCLRP scheme has a better throughput than the NADIR and EEMCCP protocols. EEMCCP scheme loses out on throughput as it focuses more on clustering and selecting a gateway node. The NADIR scheme has a lesser throughput than the SCLRP protocol since a lot of its time is spent in sending a series of control messages. Both the protocols do not have any specific encryption scheme to safeguard the data. As a result, when the data is under attack both NADIR and EEMCCP scheme loses data and time resulting in wastage of bandwidth and other node related factors. Both the schemes take time to recover in case of security issues in the network. The proposed SCLRP scheme uses EAHE scheme to safeguard the data. As a result, the transmitted data is secured well till it reaches its destination. Any attacks from external perpetrators can be thwarted because of the strong encryption applied to the data. Also, NADIR and EEMCCP do not use fuzzy rules to select the best relay node. NADIR relies on control messages and the node status, while EEMCCP is concerned with the transferring of the data to the mobile nodes towards the destination. This takes up time and reflects on the overall throughput (Fig. 11).

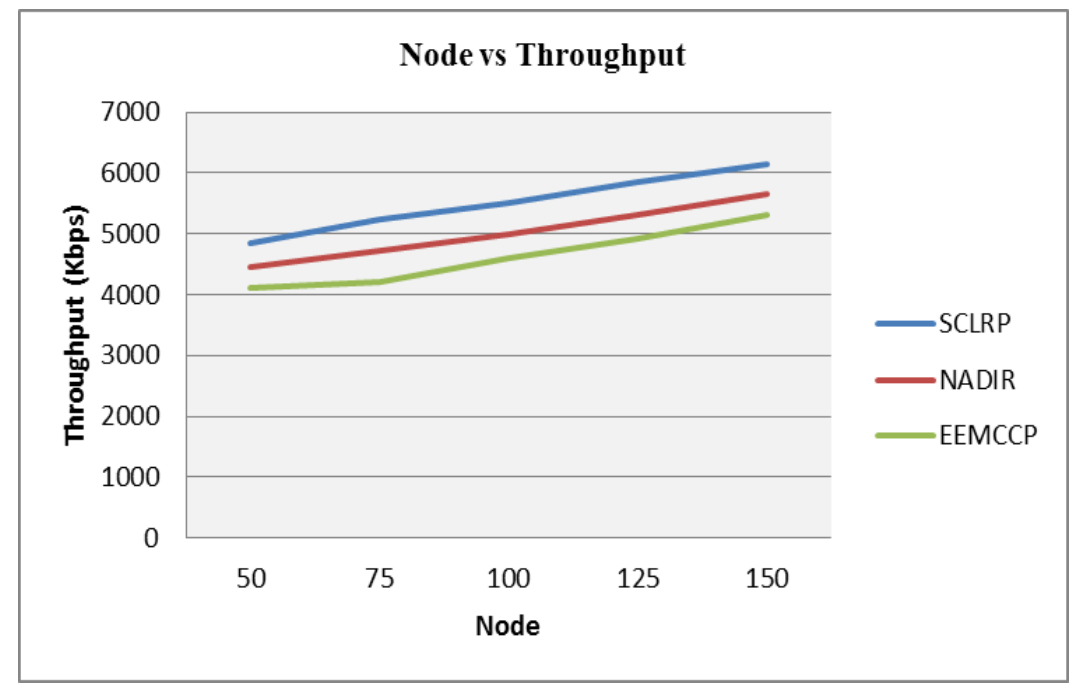

Fig.11 Throughput

\section{Conclusion and Future work}

Research is underway to identify the effective ways of communicating underwater. We have proposed Architecture for Secure Cross-Layer Routing Protocol in Underwater Acoustic Sensor Networks using Fuzzy Logic and Enhanced Algebra Homomorphic Encryption. This technique will ensure effective routing and efficient safeguarding of the transmitted data. It also focuses on conserving the energy of the nodes .The node status is studied and utility values are determined by applying fuzzy rules .Using the utility value, the next hop relay node is selected for forwarding the data towards the destination. This ensures an effective route with the best possible optimal nodes to forward the data. In addition to this, the SCLRP technique also uses an EAHE scheme to encrypt the data securely against external and unauthorized inclusion. This helps in protecting the transmitted data .the proposed SCLRP scheme is analyzed against existing techniques like NADIR and EEMCCP schemes.The SCLRP scheme exhibits a better PDR ratio and minimized delay thus leading a better overall throughput than the existing schemes. There still exist several challenges and issues to be addressed in underwater communication. For our future 
work, we are focusing on cost effective schemes that will move towards addressing the more complex challenges and issues. Research is also underway to look at the various possibilities of linking our technique with cloud based technologies. This would open up a newer arena to secure the data in more complex manner.

\section{Compliance with Ethical Standards:}

\section{Conflicts of interest:}

There are no conflicts of interest between authors.

\section{Research involving human participants and/or animals:}

There are no human participants and/or animals involved in this manuscript.

\section{Informed consent:}

There are no informed consent criteria in this manuscript.

\section{References:}

[1] John Heidemann1, Milica Stojanovic and Michele Zorzi, "Underwater sensor networks: applications, advances and challenges", Phil. Trans. R. Soc. A (2012) 370, 158-175, doi:10.1098/rsta.2011.0214.

[2] Nicopolitidis, P., Papadimitriou, G. \& Pomportsis, A. 2010 Adaptive data broadcasting in underwater wireless networks. IEEE J. Oceanic Eng. 35, 623-634. (doi:10.1109/JOE.2010.2049674)

[3] Xie, P. \& Cui, J.-H. 2007 An FEC-based reliable data transport protocol for underwater sensor networks. In Proc. 16th IEEE Int. Conf. on Computer Communications and Networks, Honolulu, HI, September 2007, pp. 747-753. IEEE

[4] R. M. Gomathi and J. Martin Leo Manickam, "Energy Efficient Static Node Selection in Underwater Acoustic Wireless Sensor Network", Wireless Personal Communications (2019) 107:709-727

[5] M. Usha, J. Sathiamoorthy, R. Ashween, Bhagavath Nishanth Ramakrishnan, "EEMCCP - A Novel Architecture Protocol Design for Efficient Data Transmission in Underwater Acoustic Wireless Sensor Network", International Journal of Computer Networks and Applications (IJCNA), DOI: 10.22247/IJCNA/2020/195674,Volume 7, Issue 2, March - April (2020),

[6] C. Namesh and B.Ramakrishnan (2015).Effective Protocol Analysis For Fixed Nodes In Under Water Sensor Network, International Journal of Oceans and Oceanography (IJOO), Volume 2, Issue 2, Feb(2015).

[7] Peleato, B. \& Stojanovic, M. 2007 Distance aware collision avoidance protocol for ad hoc underwater acoustic sensor networks. IEEE Commun. Lett. 11, 1025-1027. (doi:10.1109/LCOMM.2007.071160)

[8] Benson, B.; Li, Y.; Kastner, R.; Faunce, B.; Domond, K.; Kimball, D.; Schurgers, C. Design of a Low-Cost Underwater Acoustic Modem for Short-Range Sensor Networking Applications. In Proceeding of the 2010 IEEE OCEANS, Sydney, NSW, Australia, 24 May 2010; pp. 1-9.

[9] C. Namesh and B.Ramakrishnan (2018).MLOARP: A Multipath Low Overhead Ad-Hoc Routing Protocol for Underwater Acoustic Sensor Networks, JASC: Journal of Applied Science and Computations, Volume 5, Issue 12, December (2018).

[10] Heidemann, J.; Stojanovic, M.; Zorzi, M. Underwater sensor networks: Applications, advances and challenges. Philos. Trans. R. Soc. 2012, 370, 158-175. [CrossRef] [PubMed]

[11] J.Sathiamoorthy, Dr.B. Ramakrishnan "A competent three-tier fuzzy cluster algorithm for enhanced data transmission in

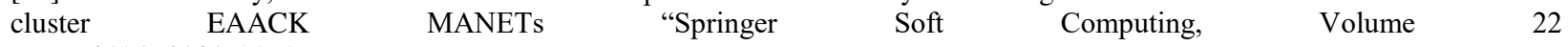
pages 6545-6565 (2018).

[12] Akyildiz, I.F.; Pompili, D.; Melodia, T. State-of-the-Art in Protocol Research for Underwater Acoustic Sensor Networks. In Proceeding of the 1st ACM International Workshop on Underwater Networks, Los Angeles, CA, USA, 25 September 2006 ; pp. $7-16$.

[13] Climent, S.; Sanchez, A.; Capella, J.V.; Meratnia, N.; Serrano, J.J. Underwater Acoustic Wireless Sensor Networks: Advances and Future Trends in Physical, MAC and Routing Layers. Sensors 2014, 14, 795-833.

[14] K. Akkaya and M. Younis, “A Survey on Routing Protocols for Wireless Sensor Networks,"Ad Hoc Networks, Vol. 3, pp. 325-349, May 2005.

[15] Namesh C., \& Ramakrishnan, B. (2018). Analysis for controlling traffic overhead in UWASN for static and moving nodes international journal of information and computing science, Volume 5, Issue 12, December 2018

[16] Toleen Jaradat, Driss Benhaddou, Manikanden Balakrishnan, Ala Al-Fuqaha. "Energy efficient cross-layer routing protocol in Wireless Sensor Networks based on fuzzy logic", 2013 9th International Wireless Communications and Mobile Computing Conference (IWCMC), 2013

[17] Radunovic, B.; Boudec, J.Y.L. Joint scheduling, power control and routing in symmetric, one dimensional, multi-hop wireless networks. In Proceedings of the Modeling and Optimization in Mobile, Ad-Hoc and Wireless Networks, SophiaAntipolis, France, 3-5 March 2003; pp. 1-11. 
[18] Tsatsanis, M.K.; Zhang, R.; Banerjee, S. Network assisted diversity for random access wireless networks. IEEE Trans. Signal Process. 2000, 48, 702-711. [CrossRef]

[19] J.Sathiamoorthy, Dr.B. Ramakrishnan "A Reliable Data Transmission in EAACK MANETs Using Hybrid Three-Tier Competent Fuzzy Cluster Algorithm "Springer Journal of Wireless Personal Communication, pp 1-20, 16 August 2017(Online) [20] Cao, M.; Raghunathan, V.; Kumar, P.R. Cross-Layer Exploitation of MAC Layer Diversity in Wireless Networks. In Proceedings of the 14th IEEE International Conference on Network Protocols, Santa Barbara, CA, USA, 12-15 November 2006; pp. 332-341.

[21] Xu, Y.; Heidemann, J.; Estrin, D. Adaptive Energy Conserving Routing for Multihop Ad Hoc Networks; Research Report 527; USC/Information Sciences Institute: Marina del Rey, CA, USA, 2000.

[22] Setton, E.; Yoo, T.; Zhu, X.Q. Cross-Layer Design of Ad-Hoc Networks for Real time Video Streaming. IEEE Wirel. Commun. 2005, 12, 59-65. [CrossRef]

[23] W. Diffie and M. Hellman, "New directions in cryptography", Information Theory, IEEE Transactions on, vol. 22, no. 6, pp. 644-54.

[24] R. L. Rivest, A. Shamir and L. Adleman, "A method for obtaining digital signatures and public-key cryptosystems", Communications of the ACM, vol. 21, no. 2, (1978), pp. 120-6.

[25] Sathiamoorthy, J., Ramakrishnan, B., \& Usha, M. (2015). A reliable and secure data transmission in CEAACK MANETs using distinct dynamic key with classified digital signature cryptographic algorithm. In IEEE international conference on computing and communications technologies (ICCCT).

[26] Sathiamoorthy, J., Ramakrishnan, B., \& Usha, M. (2015). Design of a competent broadcast algorithm for reliable transmission in CEAACK MANETs. Journal of Network Communications and Emerging Technologies, 5(1), 144-151

[27] T. Elgamal, "A public key cryptosystem and a signature scheme based on discrete logarithms", IEEE Trans Inf Theor, vol. 31, no. 4, (1985), pp. 469-72.

[28] P. Paillier, "Public-key cryptosystems based on composite degree residuosity classes", Advances in Cryptology EURPCRYPT 1999, Springer-Verlag, (1999), pp. 223-38.

[29] I. Damgrd and M. Jurik, "A generalisation, a simplification and some applications of Paillier's probabilistic public-key system", Public Key Cryptography - PKC 2001. Springer-Verlag, (2001), pp. 119-36.

[30]J.Sathiamoorthy, Dr.B. Ramakrishnan \& M. Usha "A Three Layered Peer-to-Peer Energy Efficient Protocol for Reliable and Secure Data Transmission in EAACK MANETs"Springer Journal of Wireless Personal Communication, Article First Online: 15 May 2018,

[31] J. D. Cohen and M. J. Fischer, "A robust and verifiable cryptographically secure election scheme", Proceedings of FOCS 1985, IEEE Computer Society, (1985), pp. 372-82. 32

[32] J.Sathiamoorthy, Dr.B. Ramakrishnan \& M. Usha "A Trusted Waterfall Framework Based Peer to Peer Protocol for Reliable and Energy Efficient Data Transmission in MANETs "Springer Journal of Wireless Personal Communication, Article First Online: 17 May 2018

[33] T. Okamoto and S. Uchiyama, "A new public-key cryptosystem as secure as factoring", Advances in Cryptology EUROCRYPT'98, Springer Berlin / Heidelberg, (1998), pp. 308-18.

[34] J.Sathiamoorthy, Dr.B. Ramakrishnan "STFDR: Architecture of Competent Protocol for Efficient Route Discovery and Reliable Transmission in CEAACK MANETs "Springer Journal of Wireless Personal Communication, pp 1-20, 14 August 2017(Online)

[35] J.Sathiamoorthy, Dr.B. Ramakrishnan, Usha.M "Design of a proficient hybrid protocol for efficient route discovery and secure data transmission in CEAACK MANETs" Elsevier Journal of Information Security and Applications 36 (2017)

[36] V. Vaikuntanathan, "Computing Blindfolded: new developments in fully homomorphic encryption", Proceedings of FOCS 2011. IEEE Computer Society, (2011), pp. 5-16. 
Figures

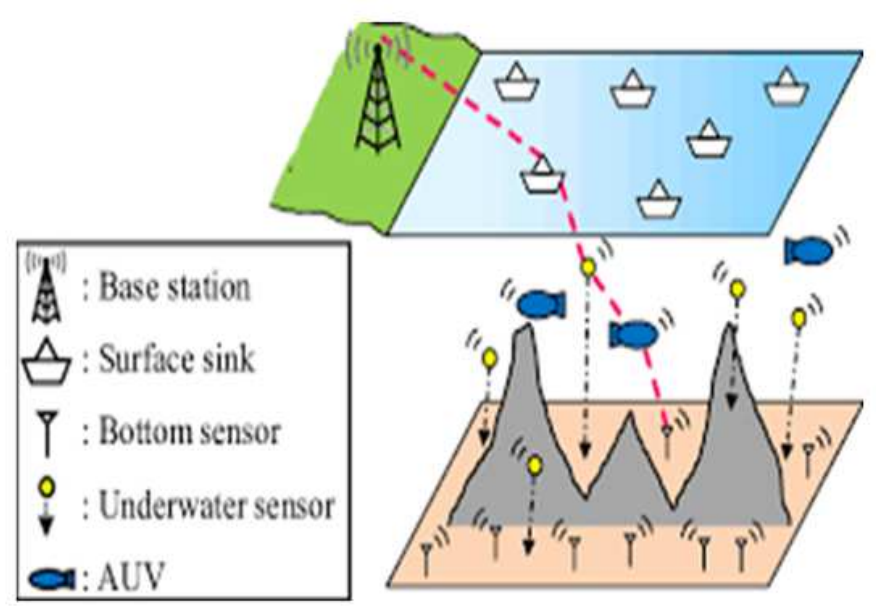

(a)

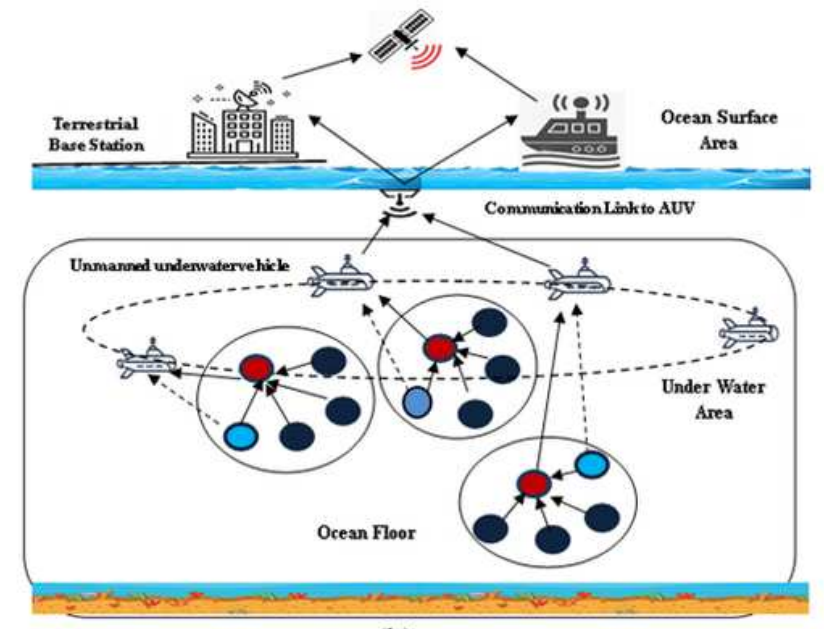

(b)

Figure 1

(a \&b) under water communication models 


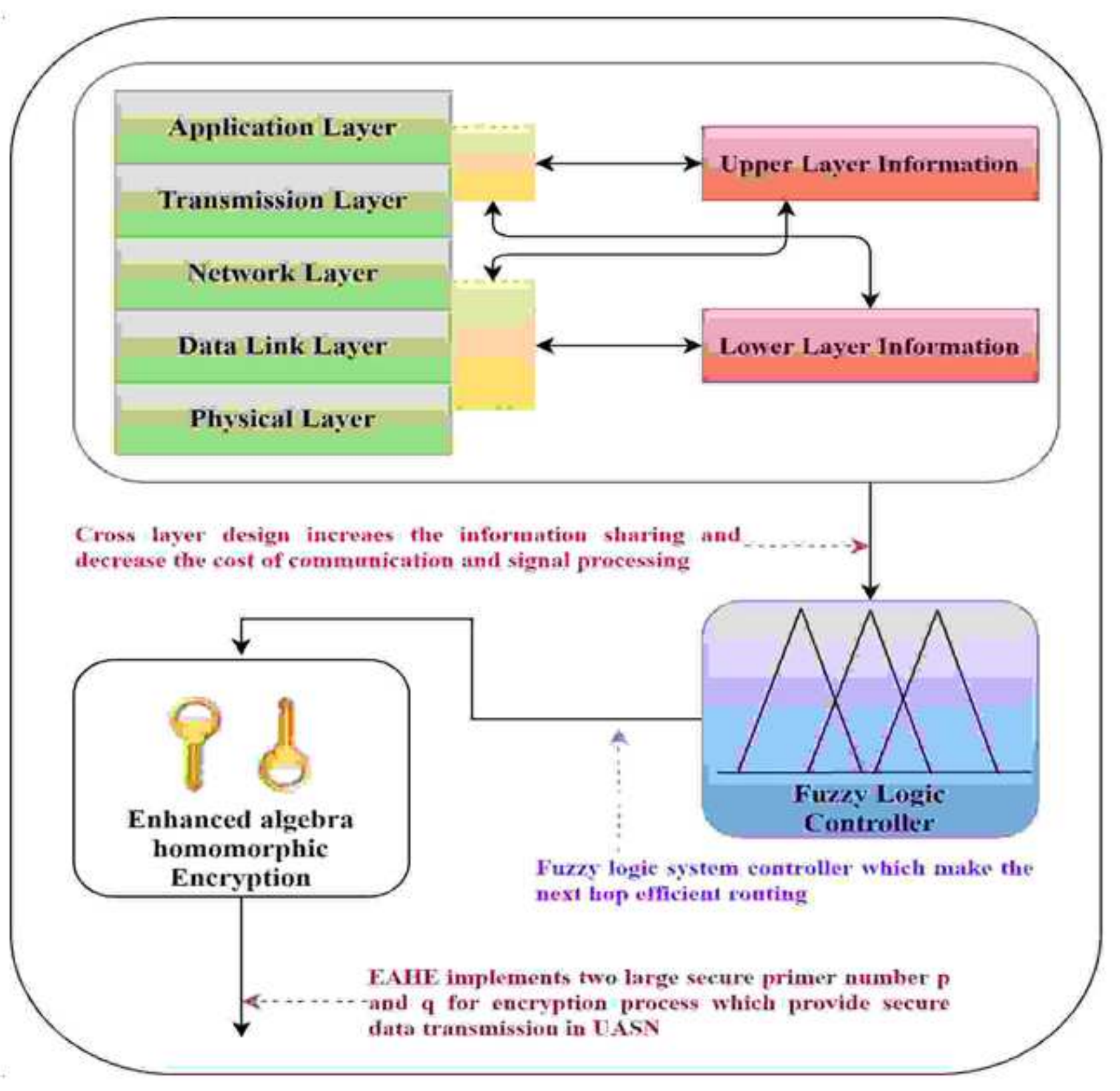

Figure 2

The architecture of proposed secure cross-layer protocol design

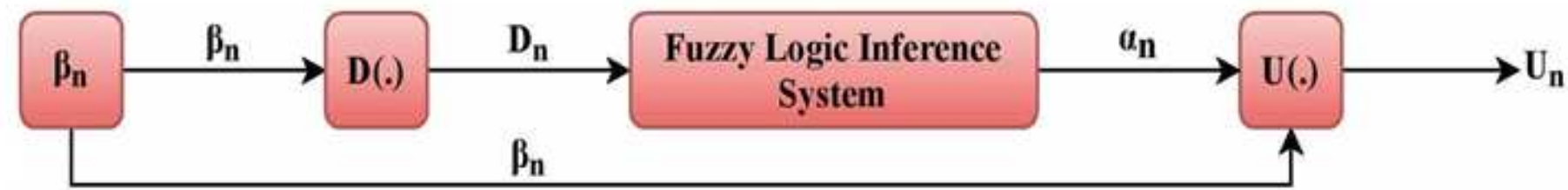

Figure 3

The principle of the SCFL 


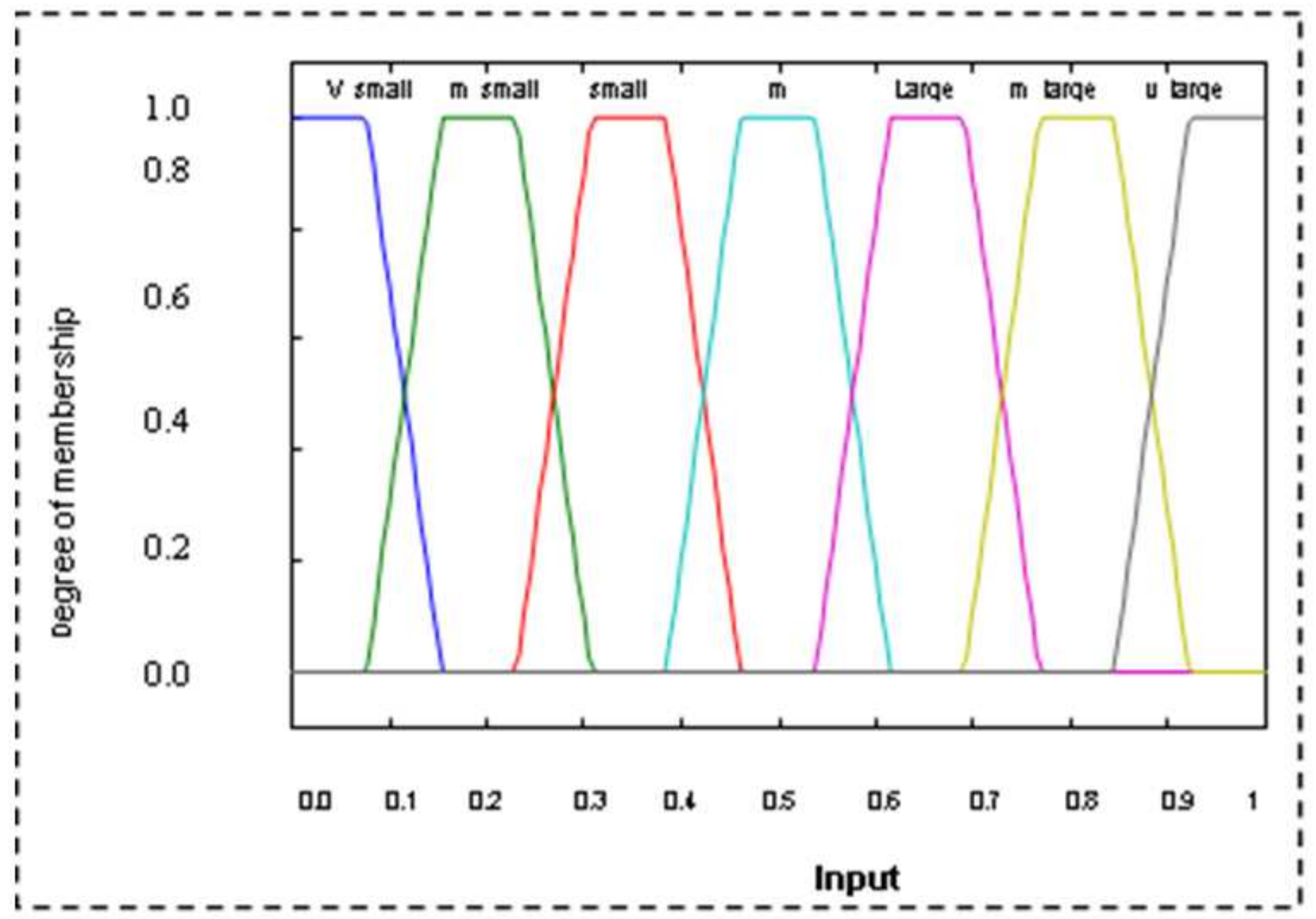

Figure 4

The membership function of input 


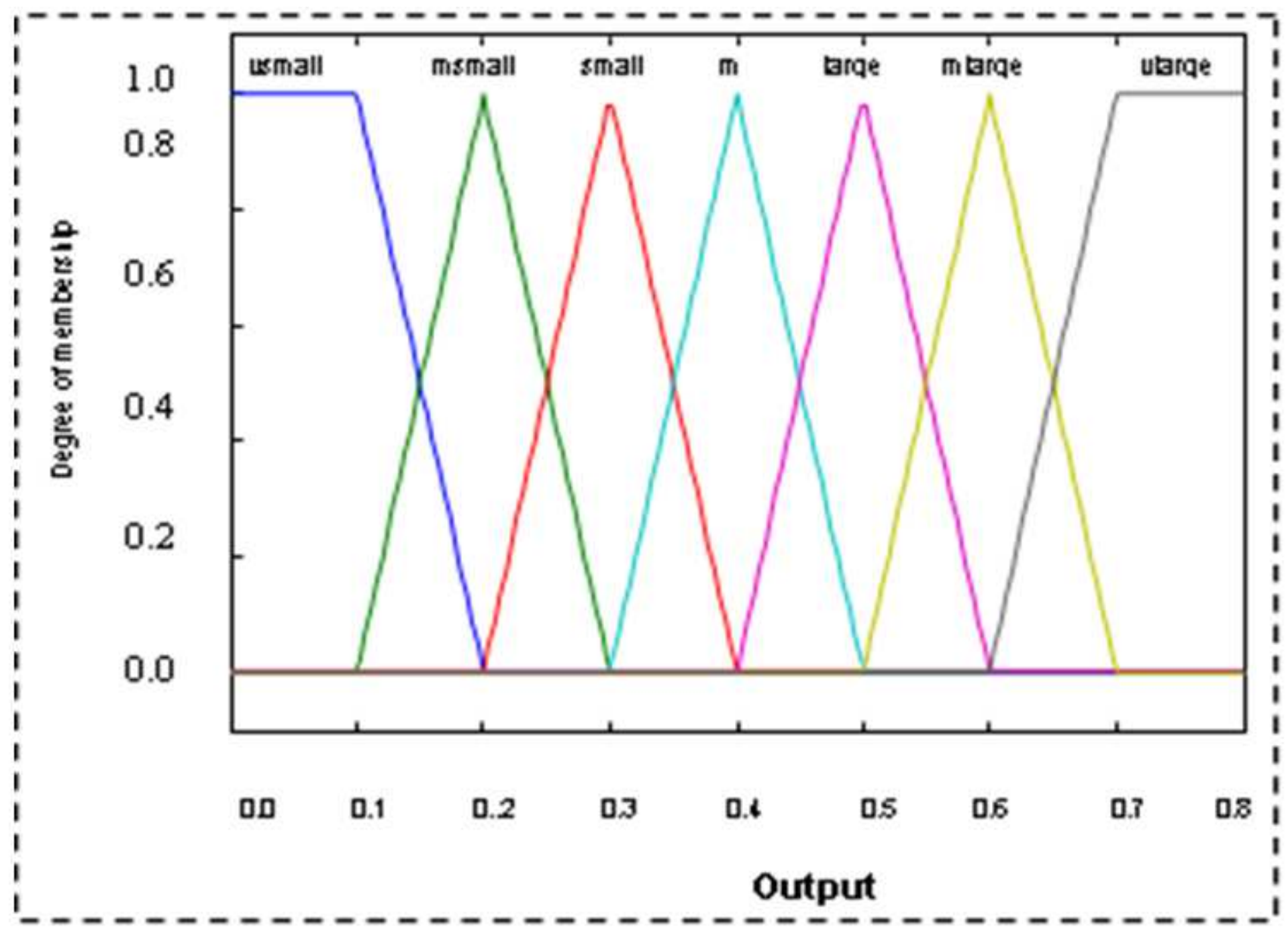

Figure 5

The membership function of output
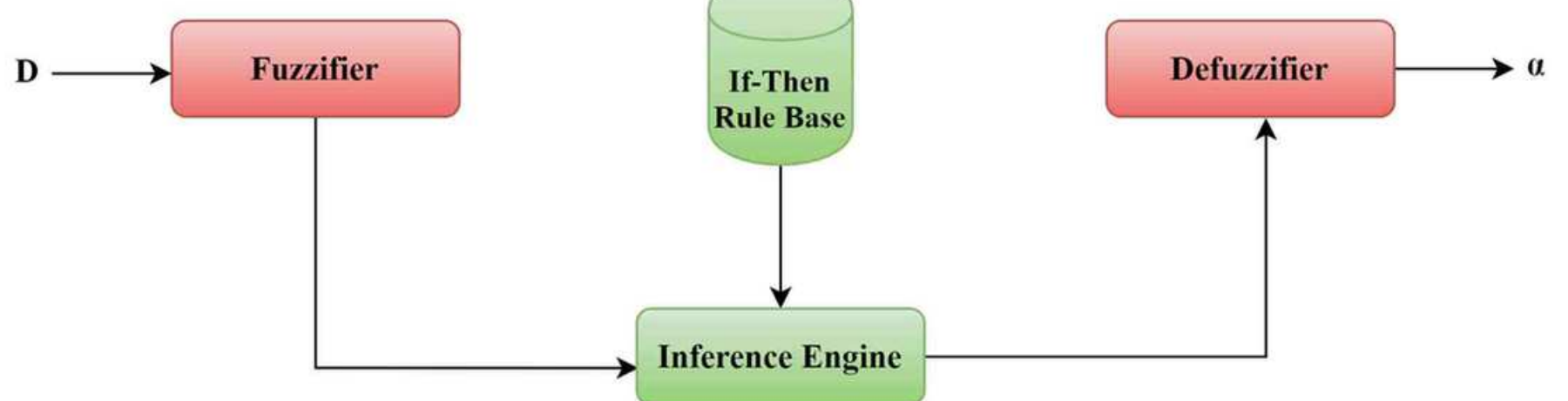

Figure 6

The Principle of Fuzzy Inference System 


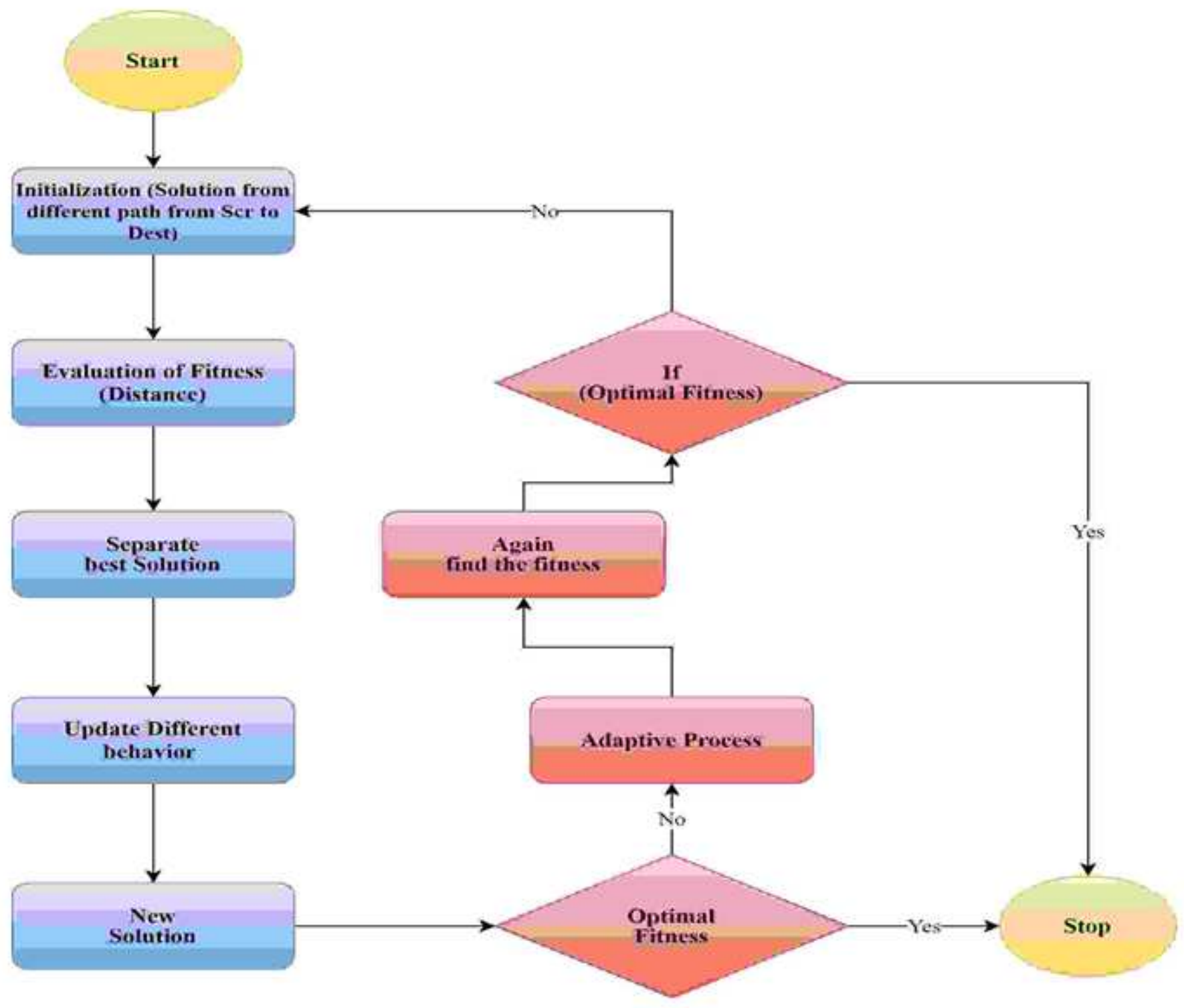

Figure 7

Data Flow Diagram for Efficient Route 


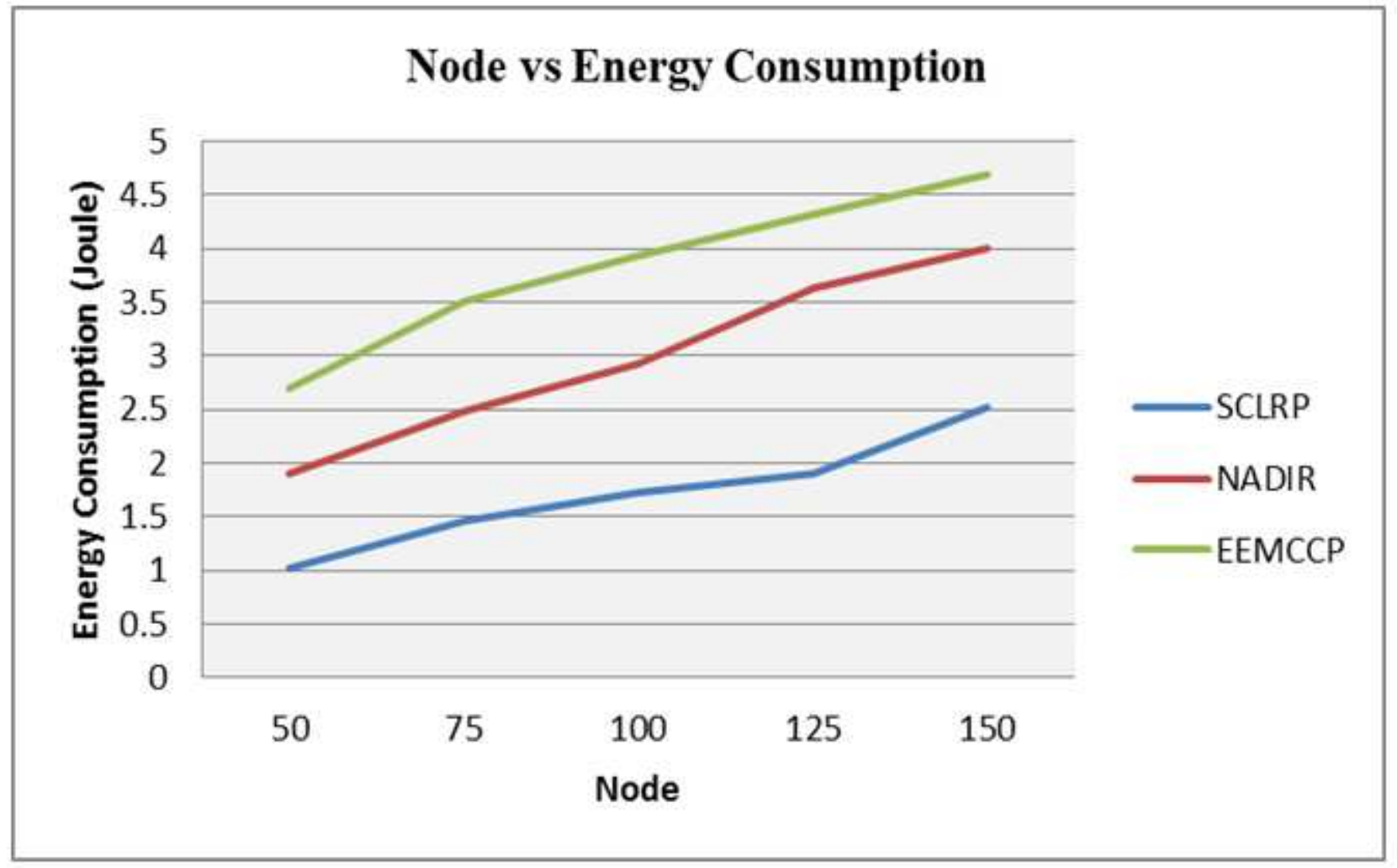

Figure 8

Energy Consumption

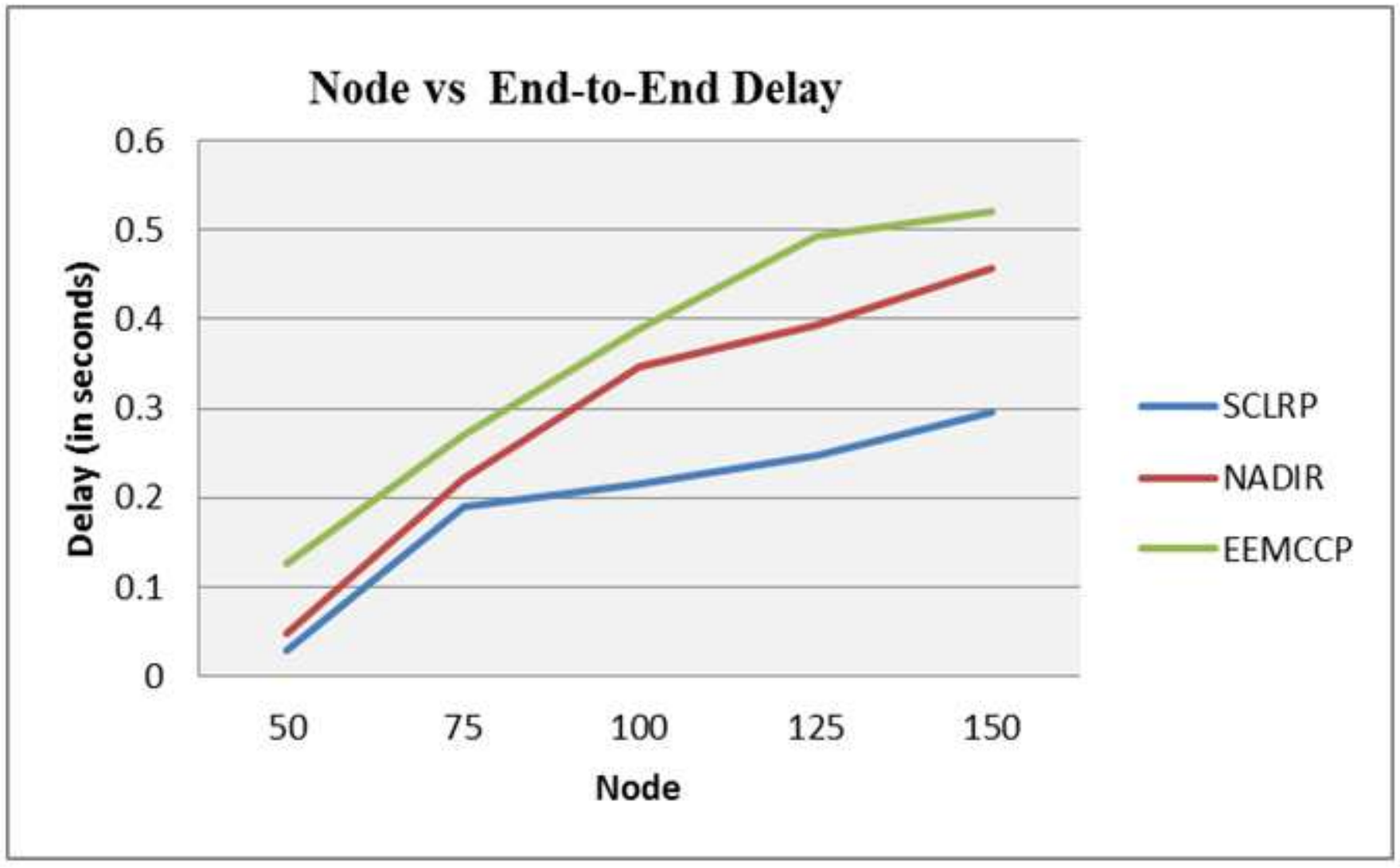


Figure 9

End to End Delay

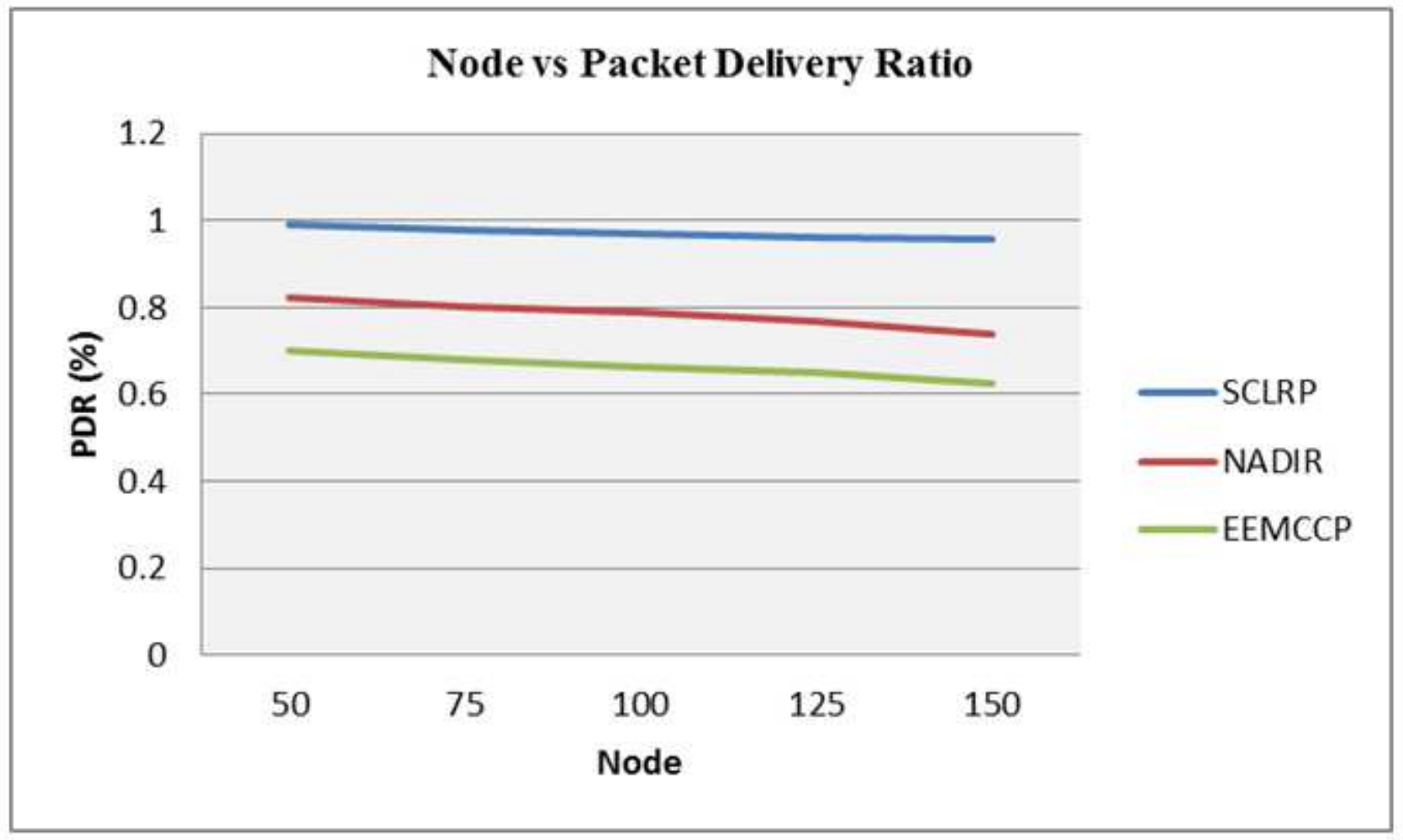

Figure 10

Packet Delivery Ratio 


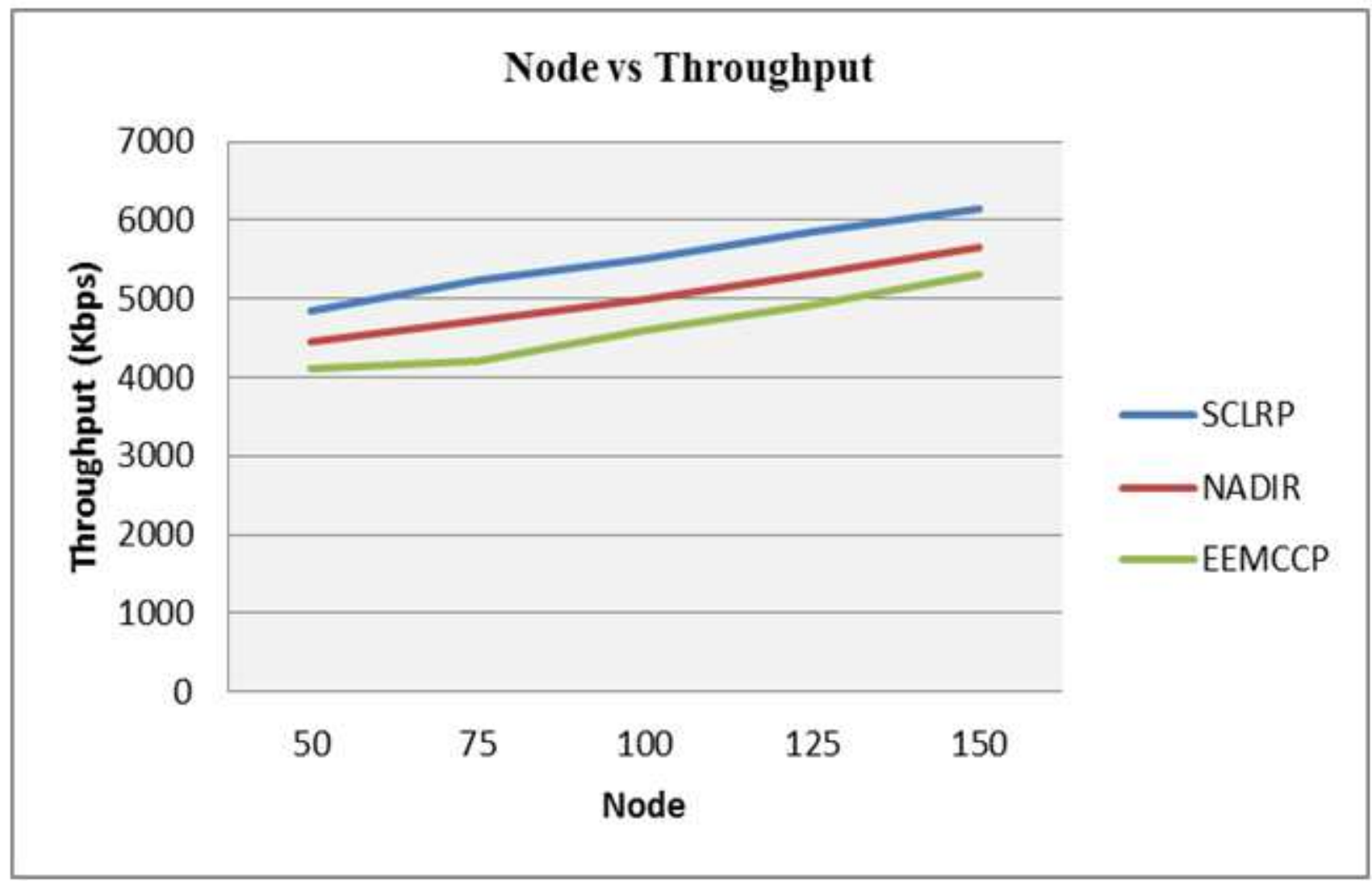

Figure 11

Throughput 